International Scientific Journal Theoretical \& Applied Science

p-ISSN: 2308-4944 (print)

e-ISSN: 2409-0085 (online)

Year: 2016

Issue: 6

Volume: 38

Published: 30.06 .2016

http://T-Science.org

SECTION 2. Applied mathematics. Mathematical modeling.
Alexandr Shevtsov candidate of technical sciences, member of PILA (USA), member of European Academy of Natural History (UK), corresponding member of the Kazakhstan National Academy of Natural Sciences,

President of International Academy TAS, Department of «Mathematics», Deputy Director on Science of faculty of information technologies, automation and telecommunications, Taraz state University named after M.Kh. Dulati, Kazakhstan $\underline{\text { Shev_AlexXXXX@mail.ru }}$

\title{
SOME ALGORITHMS BUILD THE BIFURCATION CURVES OF THE LORENZ ATTRACTOR IN MAPLE
} Maple.

Abstract: Here are investigated some problems of constructing bifurcation curves for the Lorenz attractor on

Key words: Lorenz, maple, bifurcation curves.

Language: English

Citation: Shevtsov AN (2016) SOME ALGORITHMS BUILD THE BIFURCATION CURVES OF THE LORENZ ATTRACTOR IN MAPLE. ISJ Theoretical \& Applied Science, 06 (38): 166-191.

Soi: http://s-o-i.org/1.1/TAS-06-38-33 Doi: crossef http://dx.doi.org/10.15863/TAS.2016.06.38.33

\section{Introduction}

The construction of the Lorenz model associated with the forecasting processes, stochastic attractors, turbulence, etc. In fact, the Lorentz model is too simple, not to expect stochasticity in much more complex systems. Bifurcation analysis it was shown that sometimes there is a real opportunity to build a "bifurcation tree," indicating the sequence of the various metamorphoses with the solutions in the parameter space of the system. Thus, it is possible to find a way of appearance of turbulence and build a scenario for its development.[1-12]

\section{Model}

Consider the model of the Lorenz attractor:

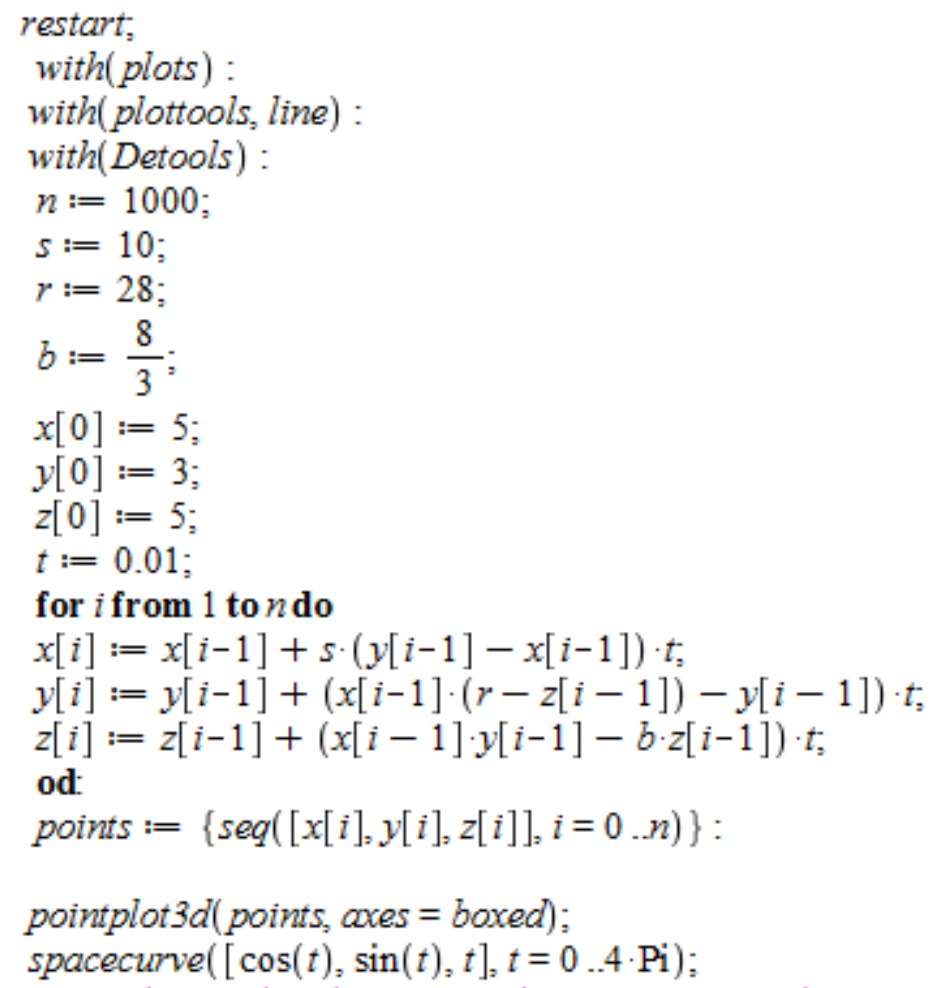




\begin{tabular}{|c|c|c|c|c|c|c|}
\hline Impact Factor: & $\begin{array}{l}\text { ISRA (India) } \\
\text { ISI (Dubai, UAE } \\
\text { GIF (Australia) } \\
\text { JIF }\end{array}$ & $\begin{array}{l}=1.344 \\
=0.829 \\
=0.564 \\
=1.500\end{array}$ & $\begin{array}{l}\text { SIS (USA) } \\
\text { PИНЦ (Russia) } \\
\text { ESJI (KZ) } \\
\text { SJIF (Morocco) }\end{array}$ & $\begin{array}{l}=0.912 \\
=0.234 \\
=1.042 \\
=2.031\end{array}$ & $\begin{array}{l}\text { ICV (Poland) } \\
\text { PIF (India) } \\
\text { IBI (India) }\end{array}$ & $\begin{array}{l}=6.630 \\
=1.940 \\
=4.260\end{array}$ \\
\hline
\end{tabular}

$$
\begin{aligned}
n & :=1000 \\
s & :=10 \\
r & :=28 \\
b & :=\frac{8}{3} \\
x_{0} & :=5 \\
y_{0} & :=3 \\
z_{0} & :=5 \\
t & :=0.01
\end{aligned}
$$

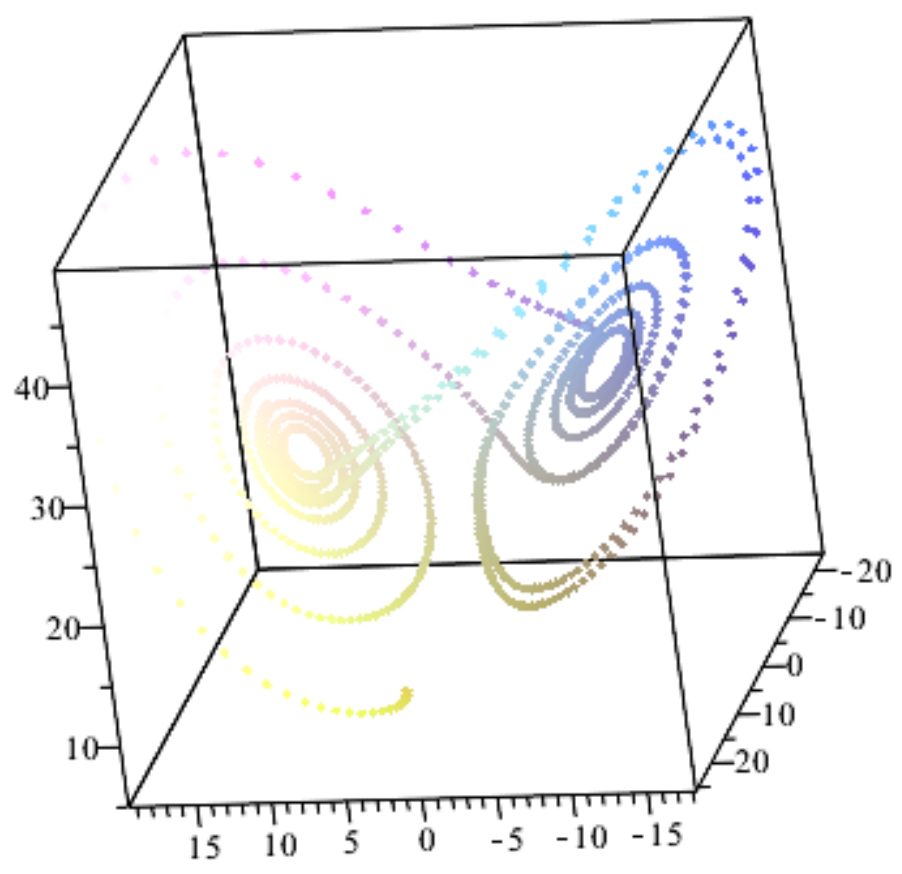

Figure 1 - Model of the Lorenz attractor.

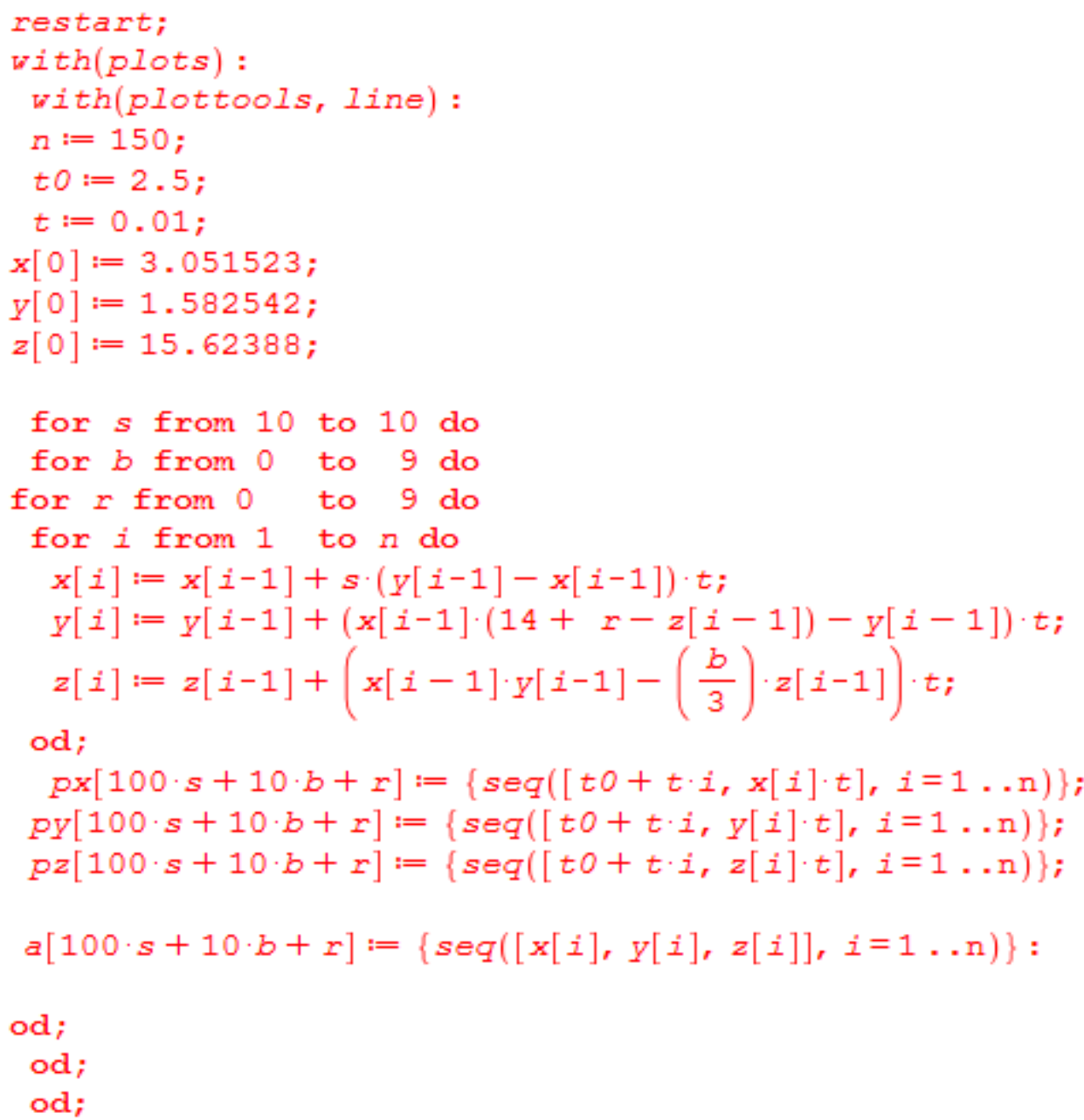




\begin{tabular}{l|lrl|l|ll} 
& ISRA (India) & $=\mathbf{1 . 3 4 4}$ & SIS (USA) & $=\mathbf{0 . 9 1 2}$ & ICV (Poland) & $=\mathbf{6 . 6 3 0}$ \\
Impact Factor: & ISI (Dubai, UAE) $=\mathbf{0 . 8 2 9}$ & PUHU (Russia) $=\mathbf{0 . 2 3 4}$ & PIF (India) & $=\mathbf{1 . 9 4 0}$ \\
& GIF (Australia) & $\mathbf{0 . 5 6 4}$ & ESJI (KZ) & $=\mathbf{1 . 0 4 2}$ & IBI (India) & $\mathbf{4 . 2 6 0}$
\end{tabular}

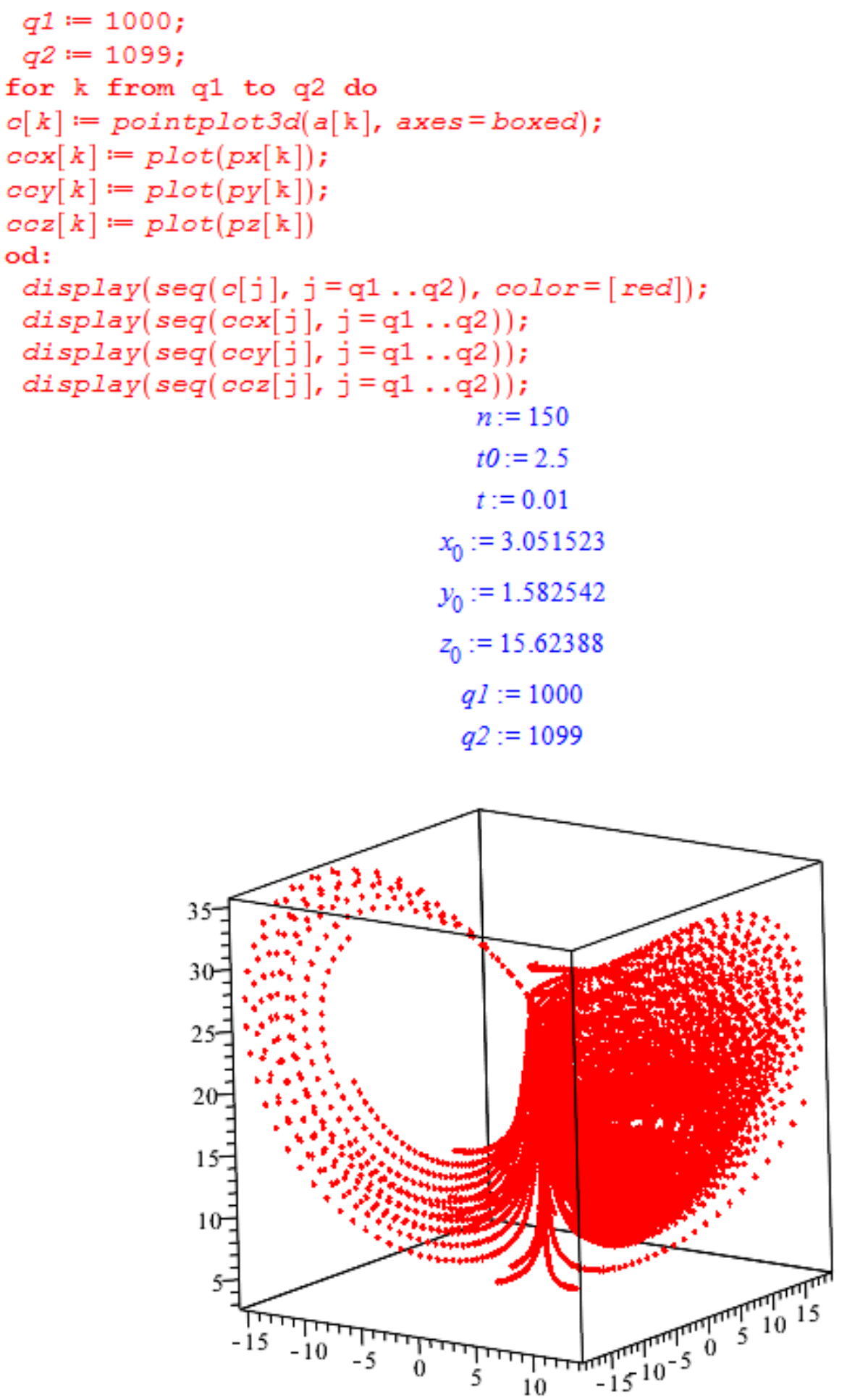

Figure 2 - Model of the Lorenz attractor, $r=0 . .9, b=0 . .9, s=10$. 


\begin{tabular}{l|lrl|l|ll} 
& ISRA (India) & $=\mathbf{1 . 3 4 4}$ & SIS (USA) & $=\mathbf{0 . 9 1 2}$ & ICV (Poland) & $=\mathbf{6 . 6 3 0}$ \\
Impact Factor: & ISI (Dubai, UAE) $=\mathbf{0 . 8 2 9}$ & PUHU (Russia) $=\mathbf{0 . 2 3 4}$ & PIF (India) & $=\mathbf{1 . 9 4 0}$ \\
& GIF (Australia) & $\mathbf{0 . 5 6 4}$ & ESJI (KZ) & $=\mathbf{1 . 0 4 2}$ & IBI (India) & $=\mathbf{4 . 2 6 0}$
\end{tabular}

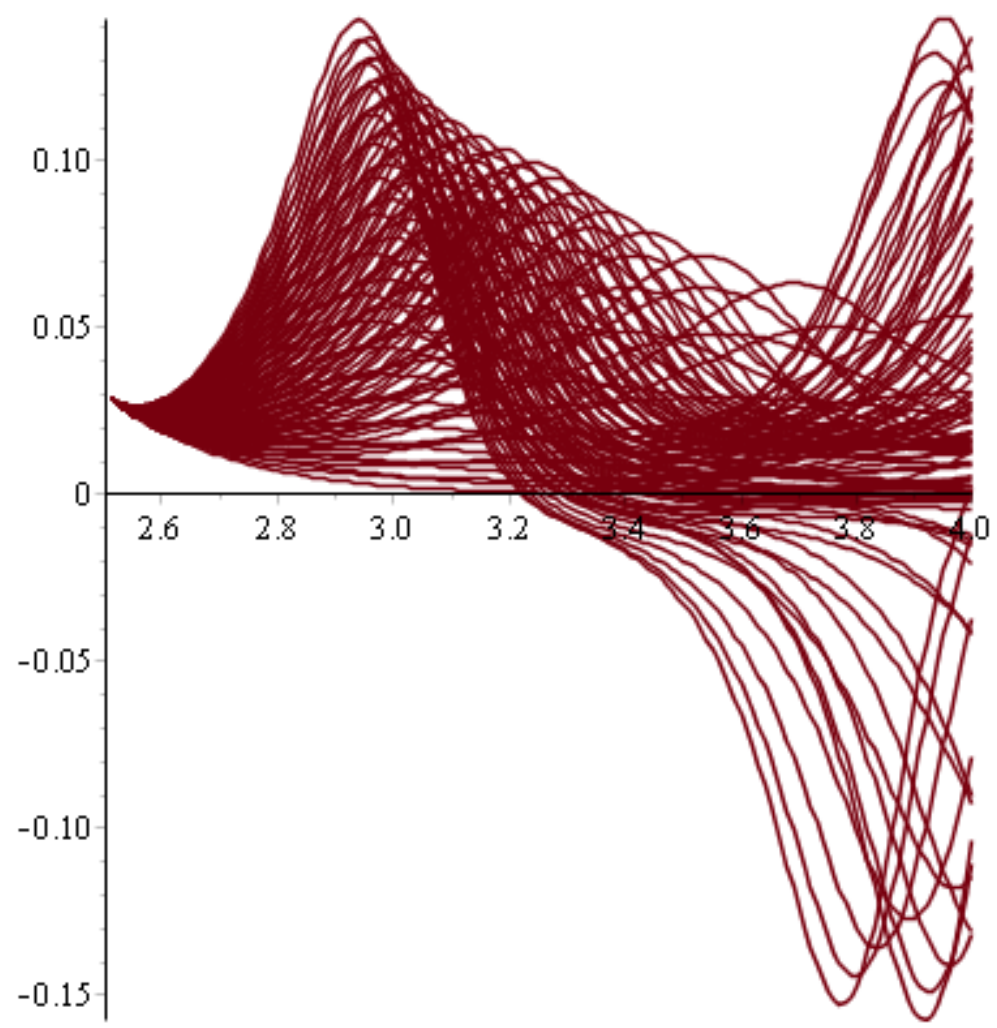

Figure 3 - Bifurcation of the function $x[t]$.

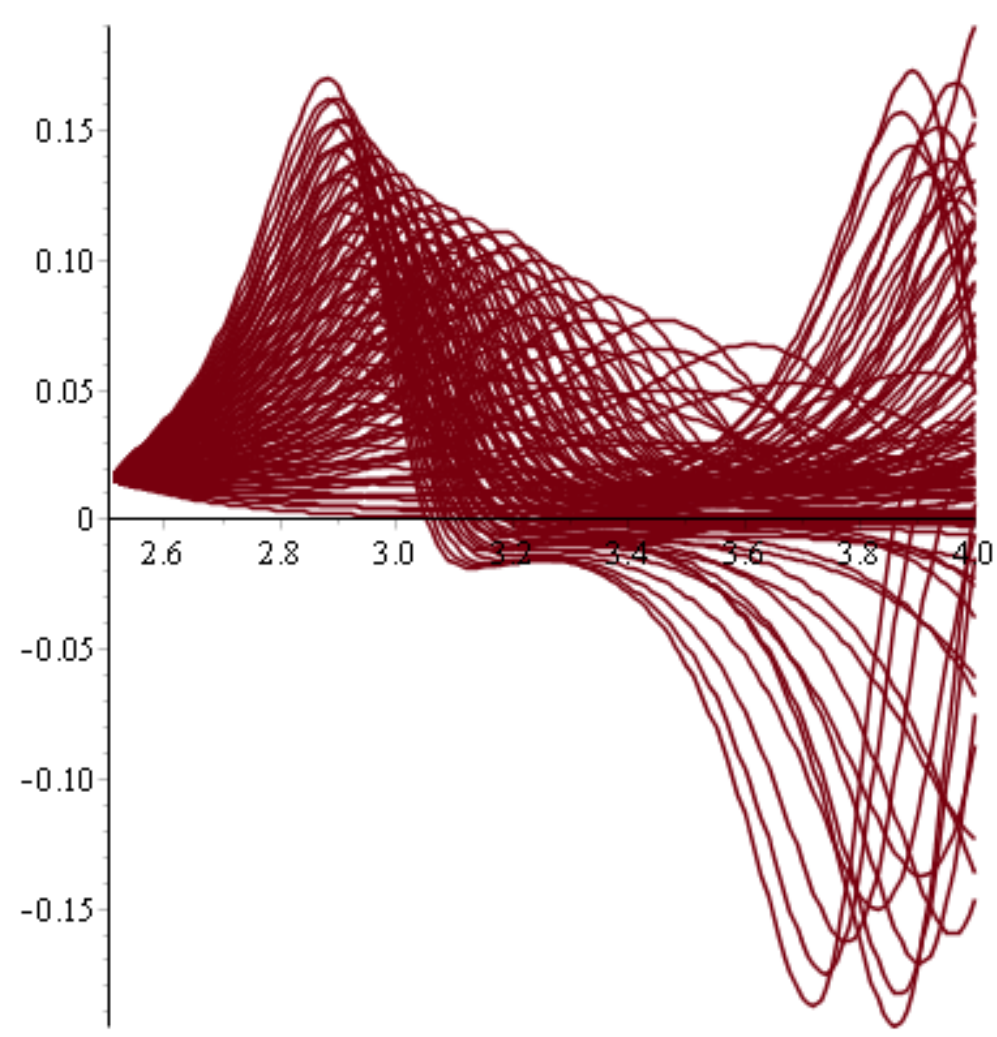

Figure 4 - Bifurcation of the function $y[t]$. 


\begin{tabular}{l|lrl|l|ll} 
& ISRA (India) & $=\mathbf{1 . 3 4 4}$ & SIS (USA) & $=\mathbf{0 . 9 1 2}$ & ICV (Poland) & $=\mathbf{6 . 6 3 0}$ \\
Impact Factor: & ISI (Dubai, UAE) $=\mathbf{0 . 8 2 9}$ & PUHU (Russia) $=\mathbf{0 . 2 3 4}$ & PIF (India) & $=\mathbf{1 . 9 4 0}$ \\
& GIF (Australia) & $\mathbf{0 . 5 6 4}$ & ESJI (KZ) & $=\mathbf{1 . 0 4 2}$ & IBI (India) & $=\mathbf{4 . 2 6 0}$
\end{tabular}

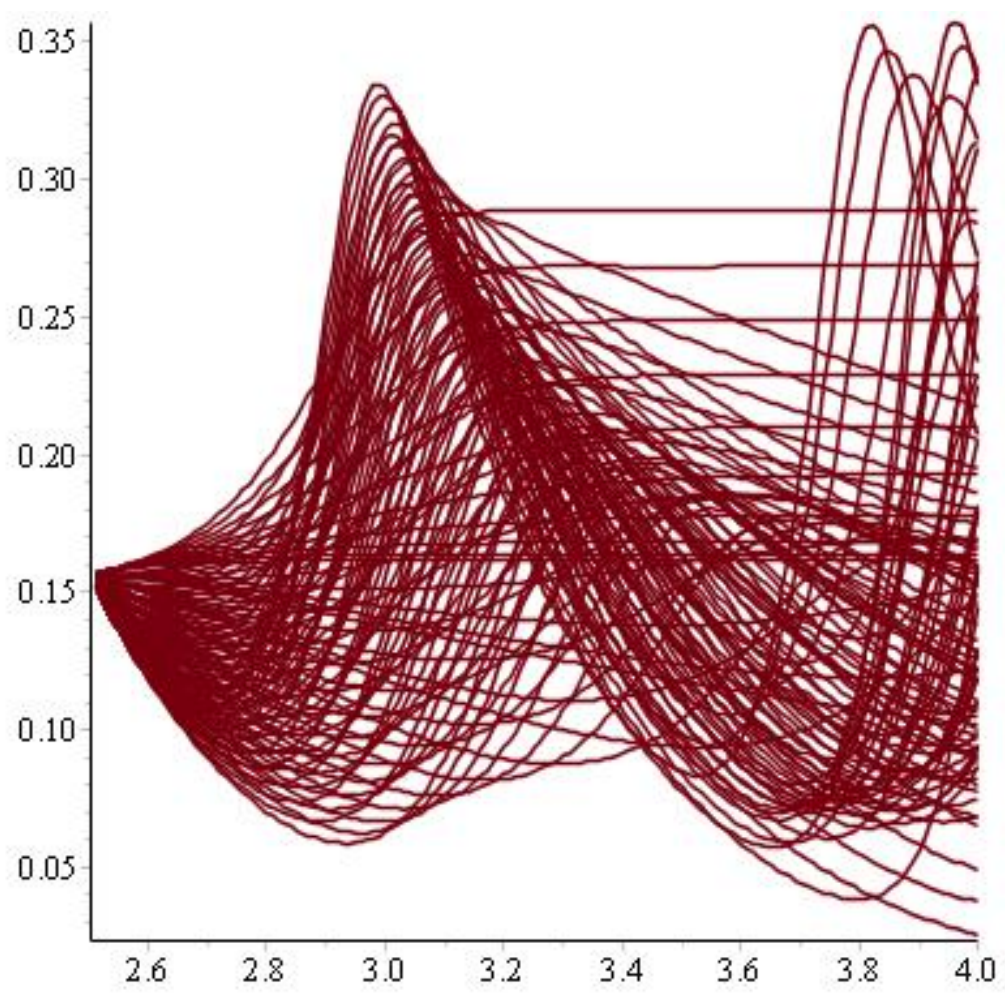

Figure 5 - Bifurcation of the function $\mathrm{z}[\mathrm{t}]$.

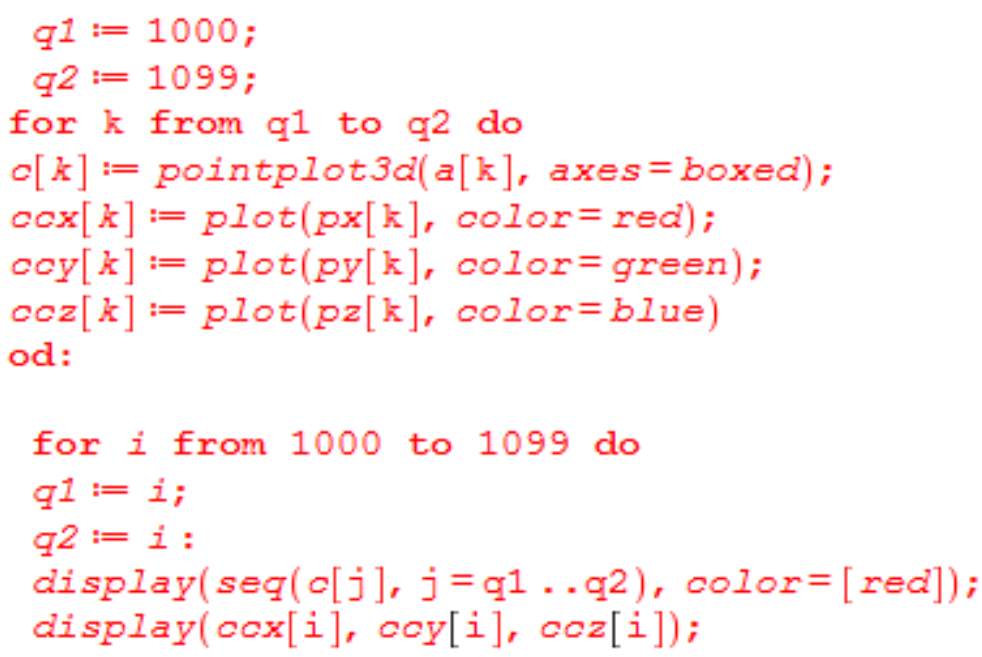

od; 


\begin{tabular}{l|lr|ll|ll} 
& ISRA (India) & $=\mathbf{1 . 3 4 4}$ & SIS (USA) & $=\mathbf{0 . 9 1 2}$ & ICV (Poland) & $=\mathbf{6 . 6 3 0}$ \\
Impact Factor: & ISI (Dubai, UAE) & $=\mathbf{0 . 8 2 9}$ & PUH (Russia) & $=\mathbf{0 . 2 3 4}$ & PIF (India) & $=\mathbf{1 . 9 4 0}$ \\
& GIF (Australia) & $=\mathbf{0 . 5 6 4}$ & ESJI (KZ) & $=\mathbf{1 . 0 4 2}$ & IBI (India) & $=\mathbf{4 . 2 6 0}$ \\
& JIF & $=\mathbf{1 . 5 0 0}$ & SJIF (Morocco) & $\mathbf{2 . 0 3 1}$ & & \\
\hline
\end{tabular}

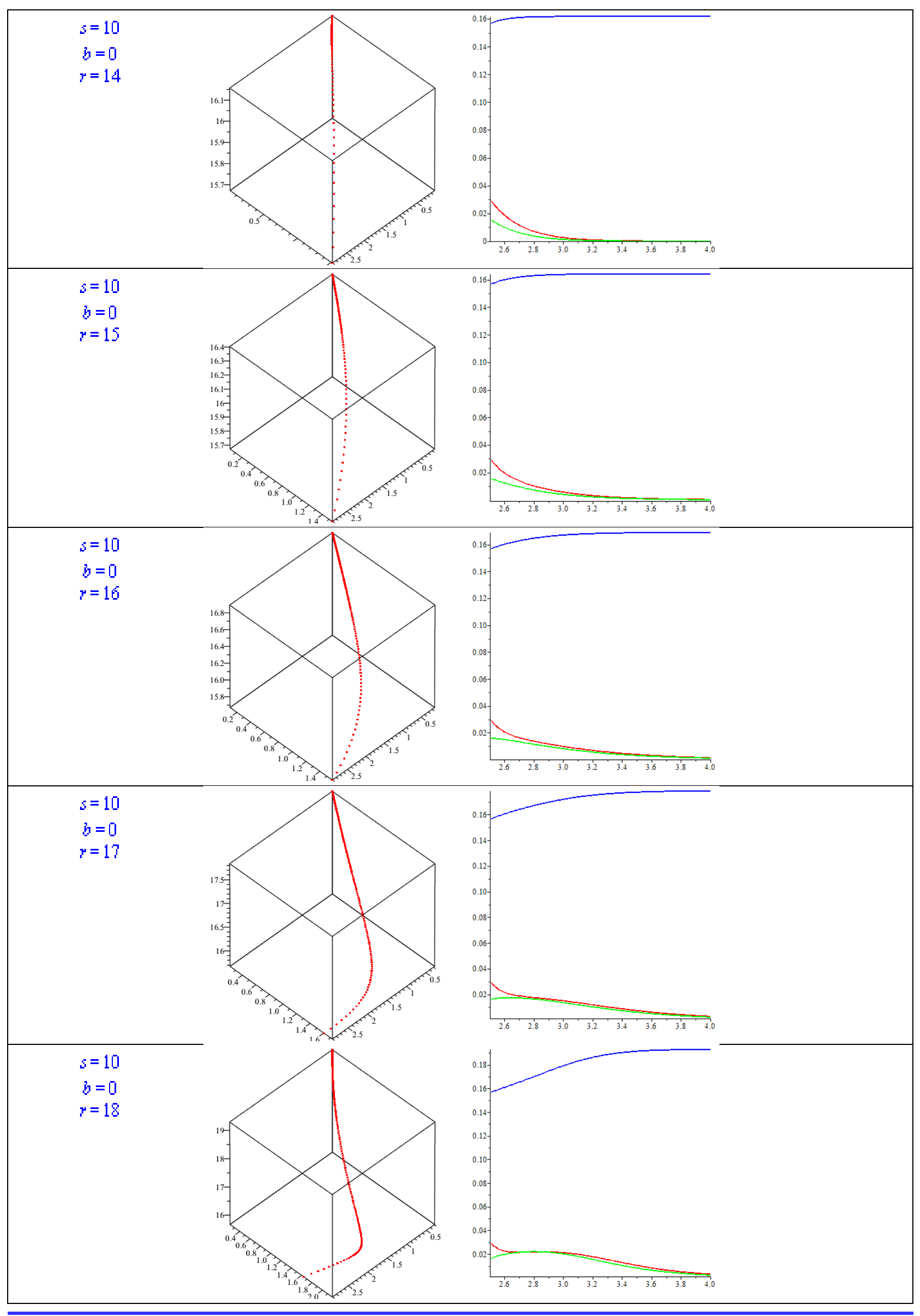




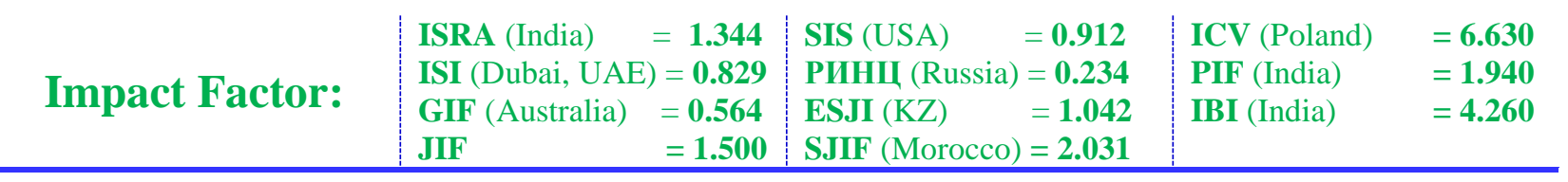

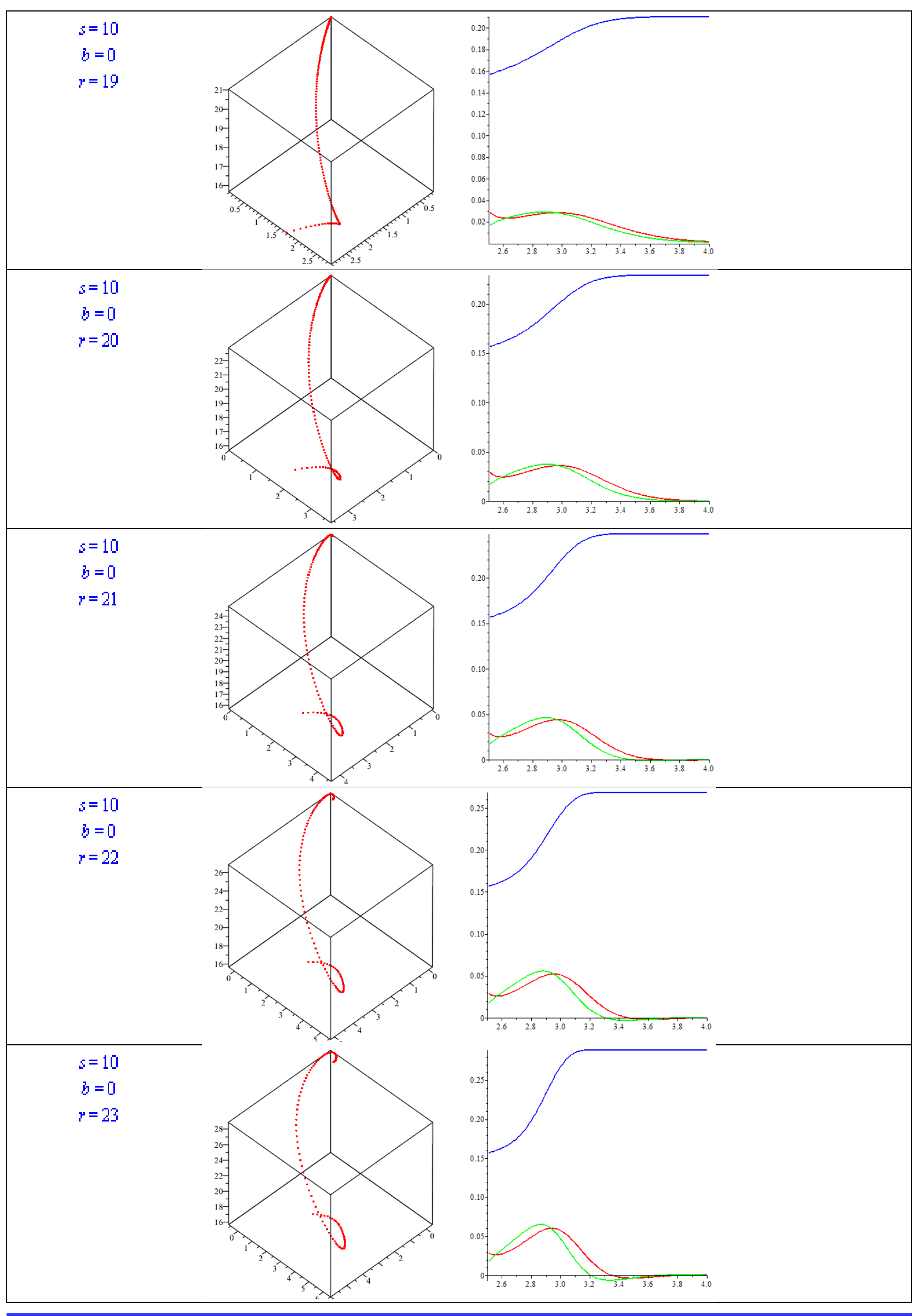




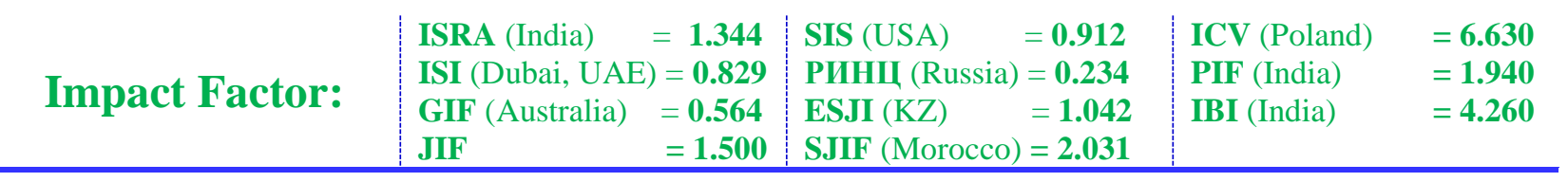

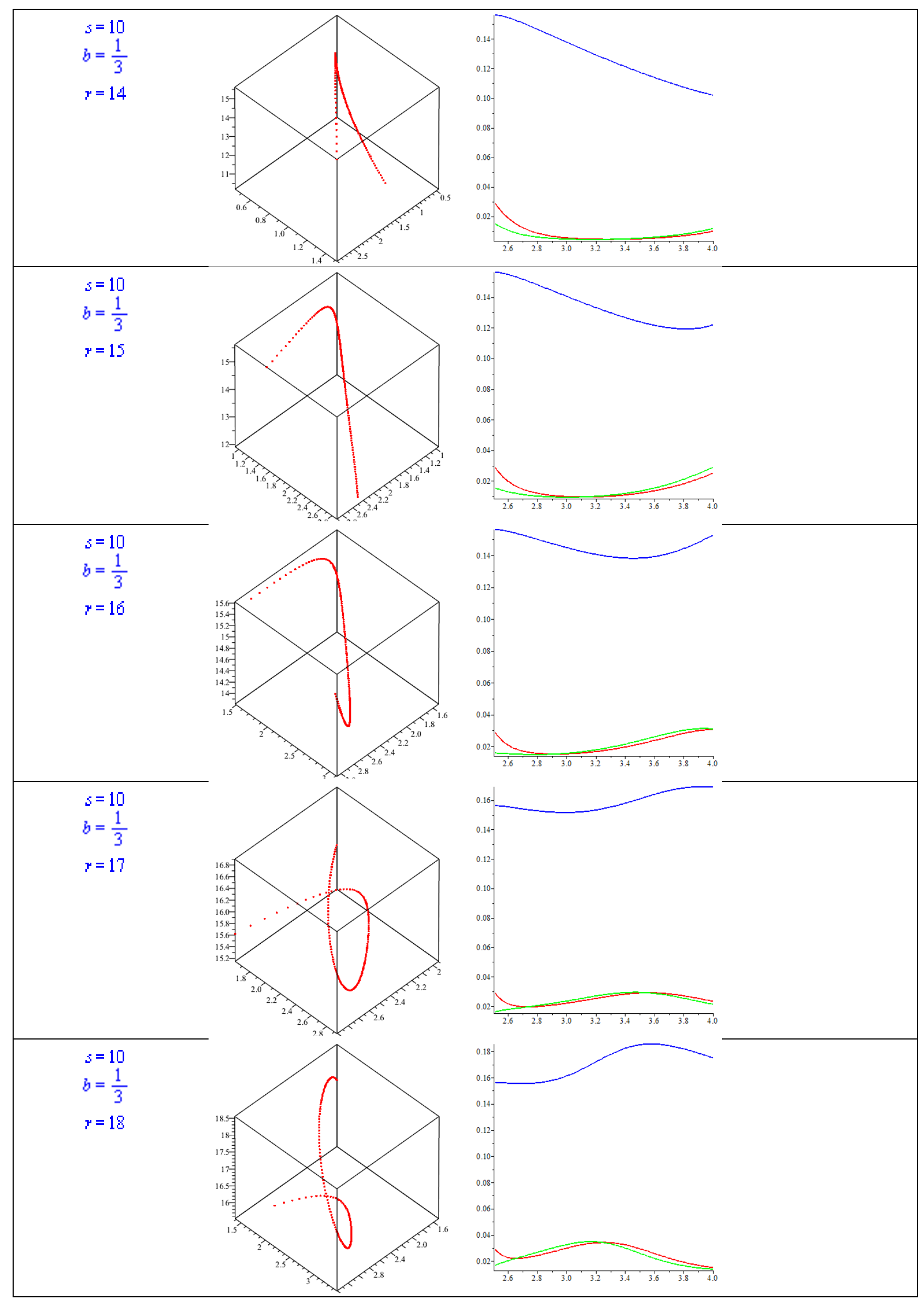




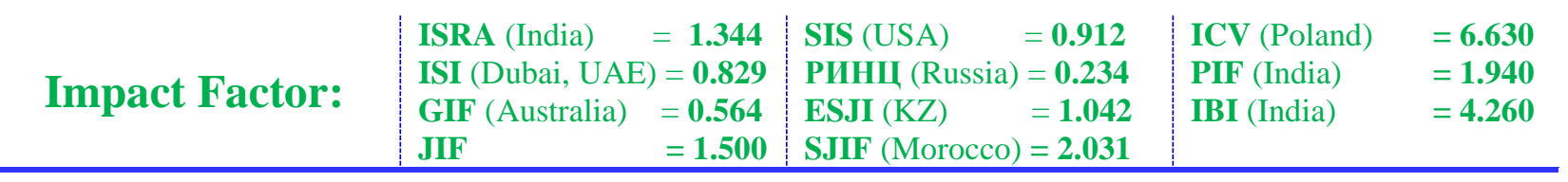

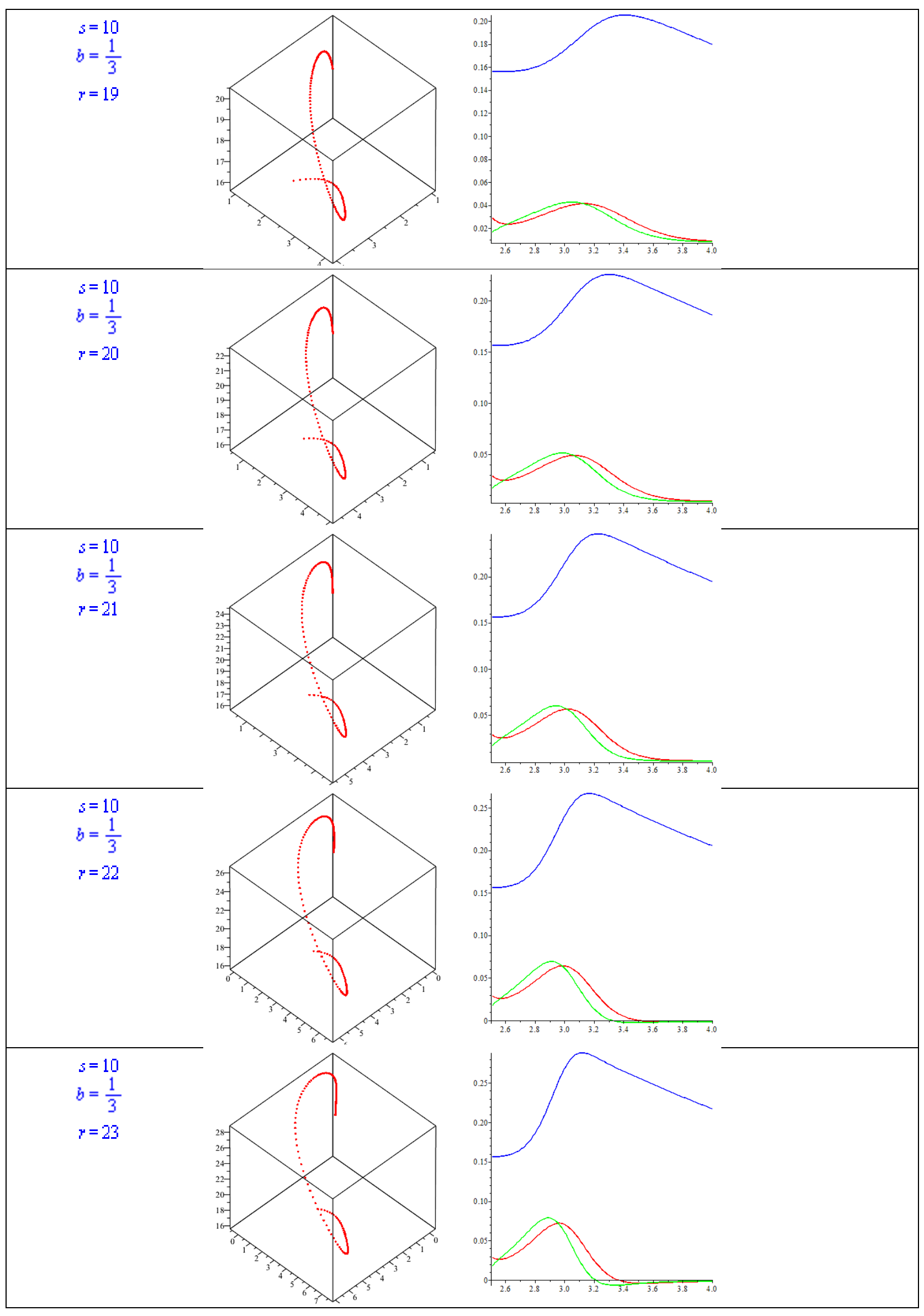




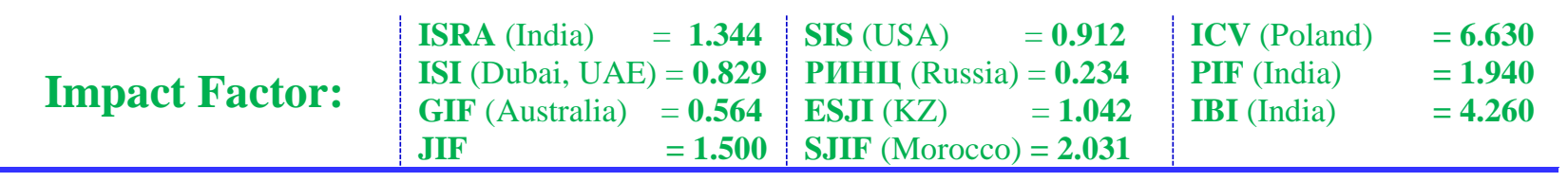

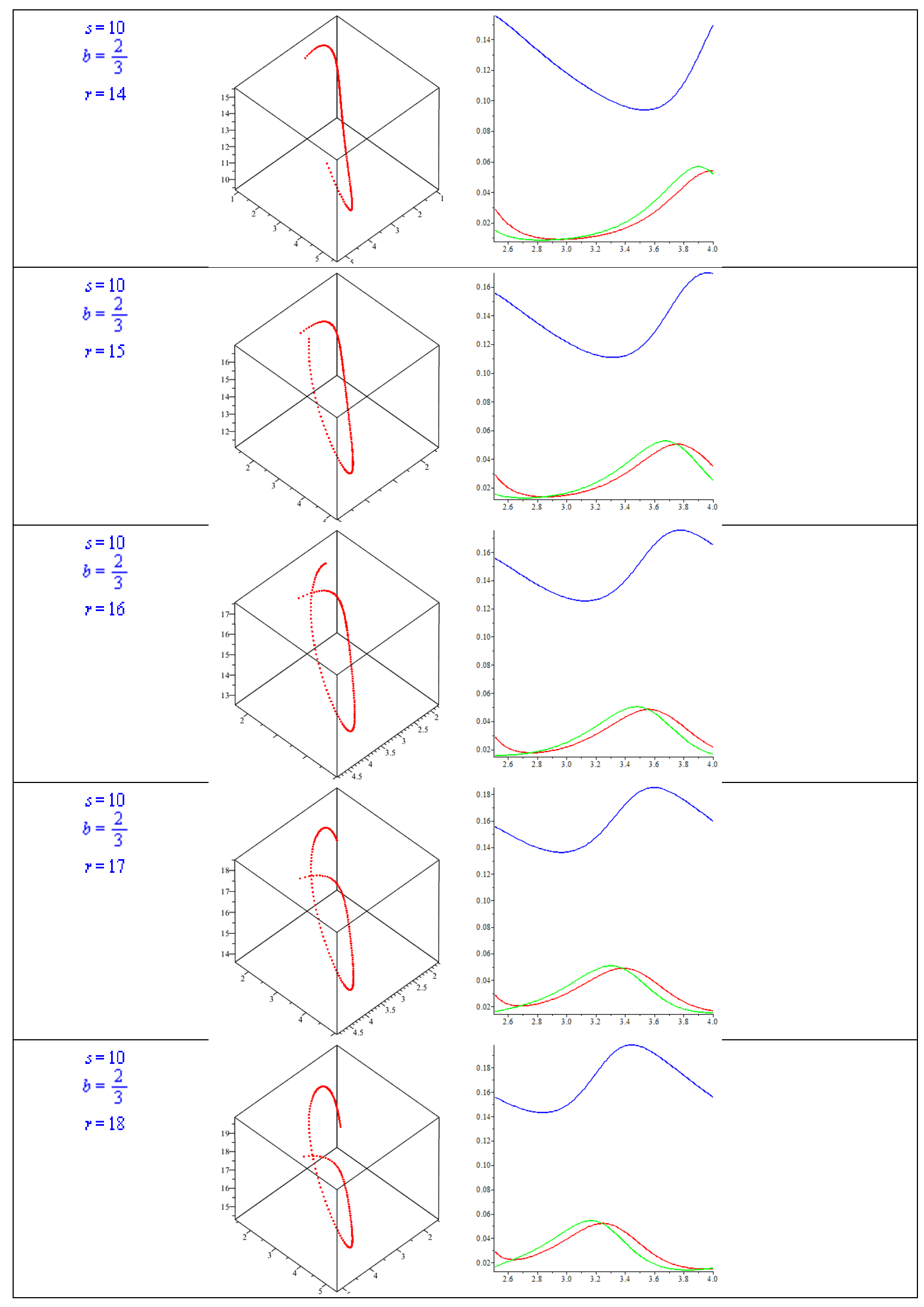




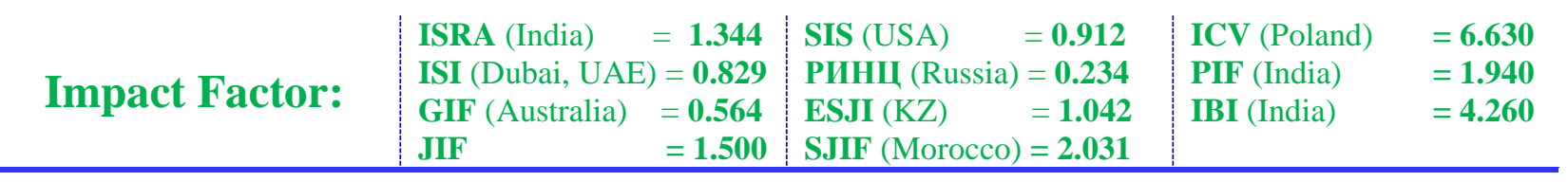

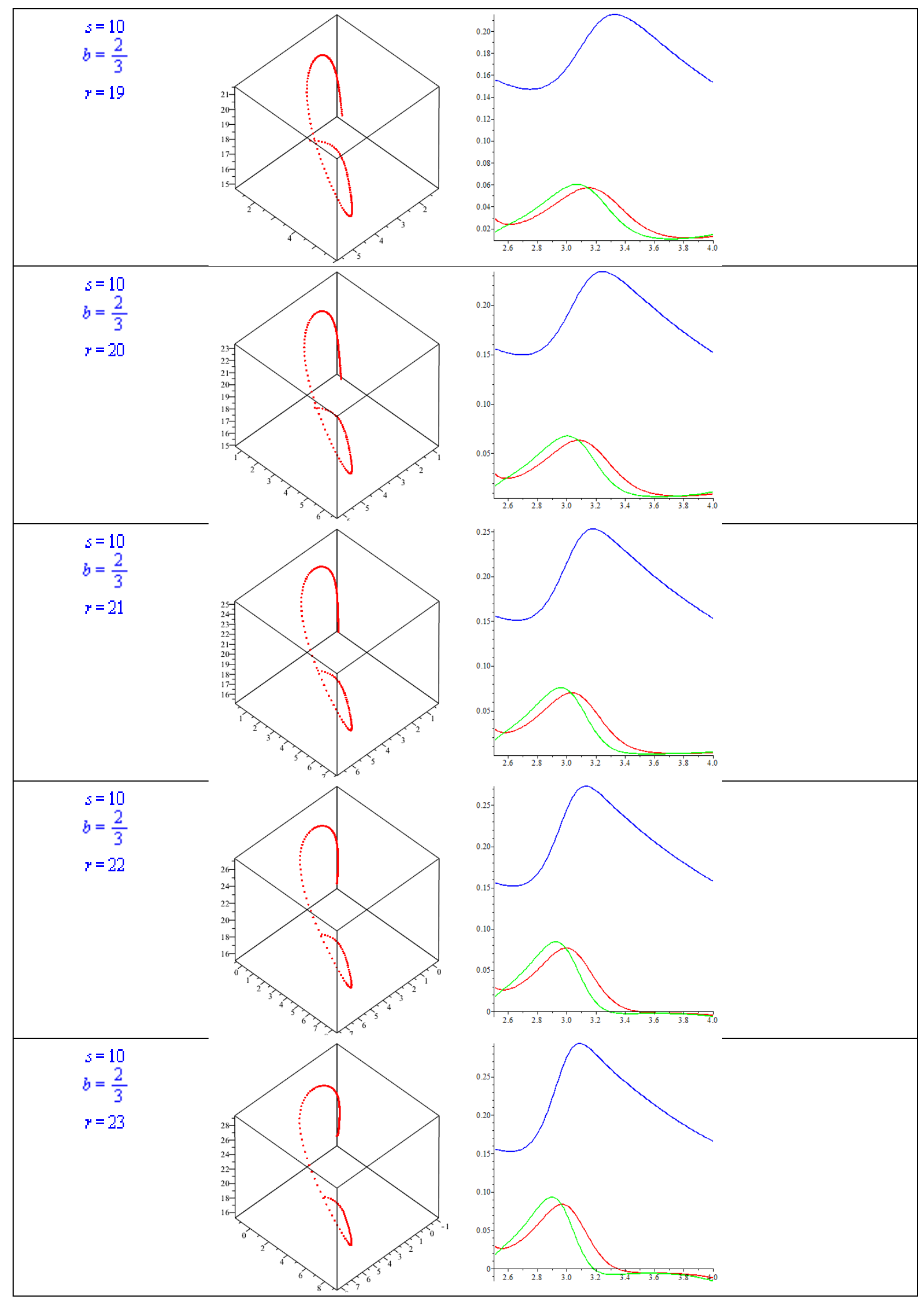




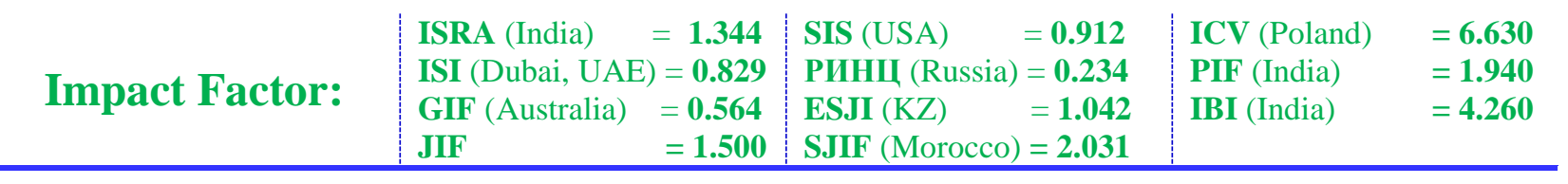

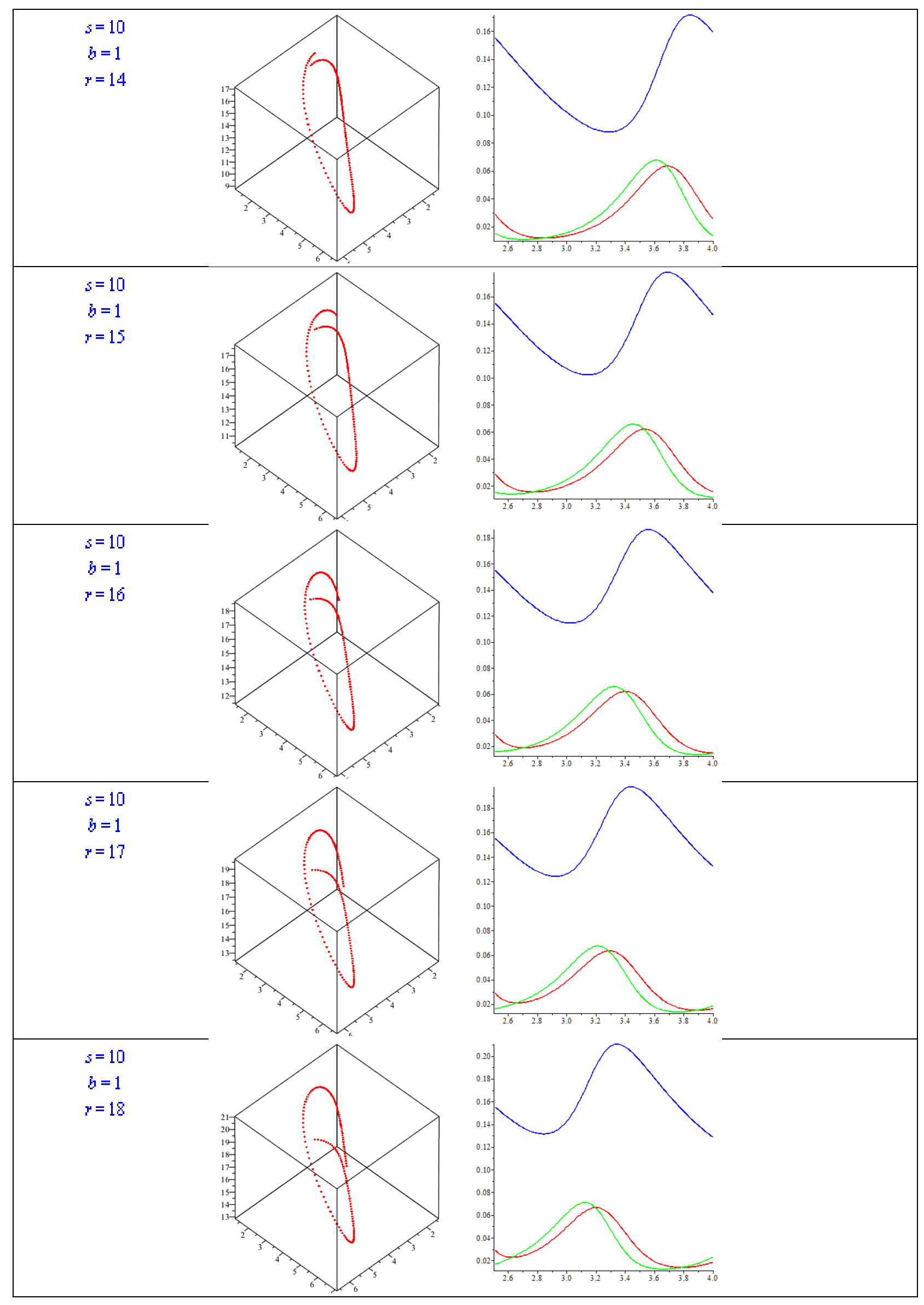




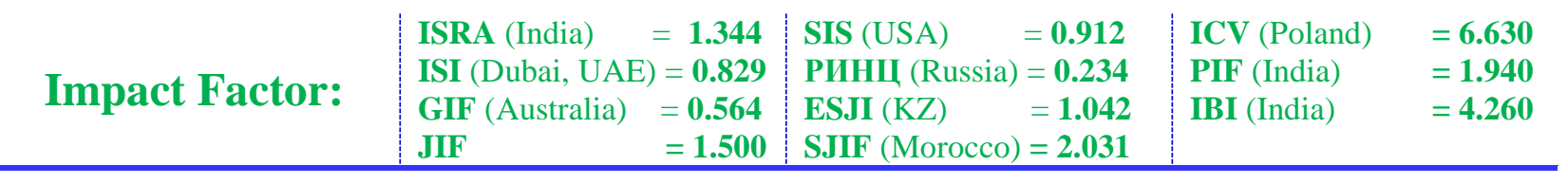

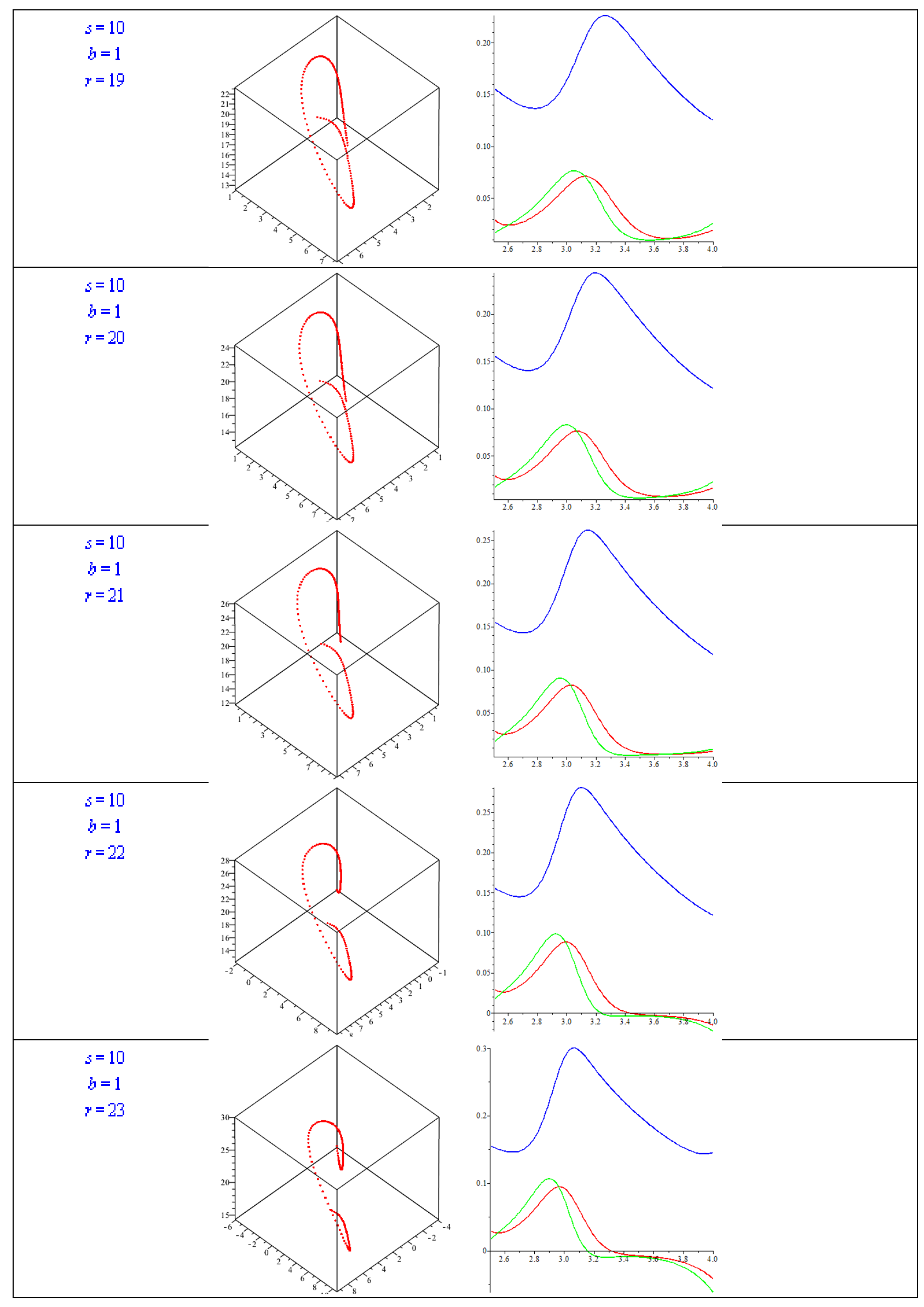




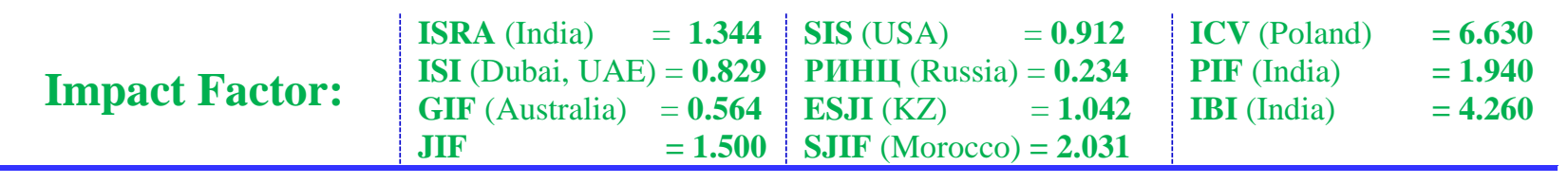

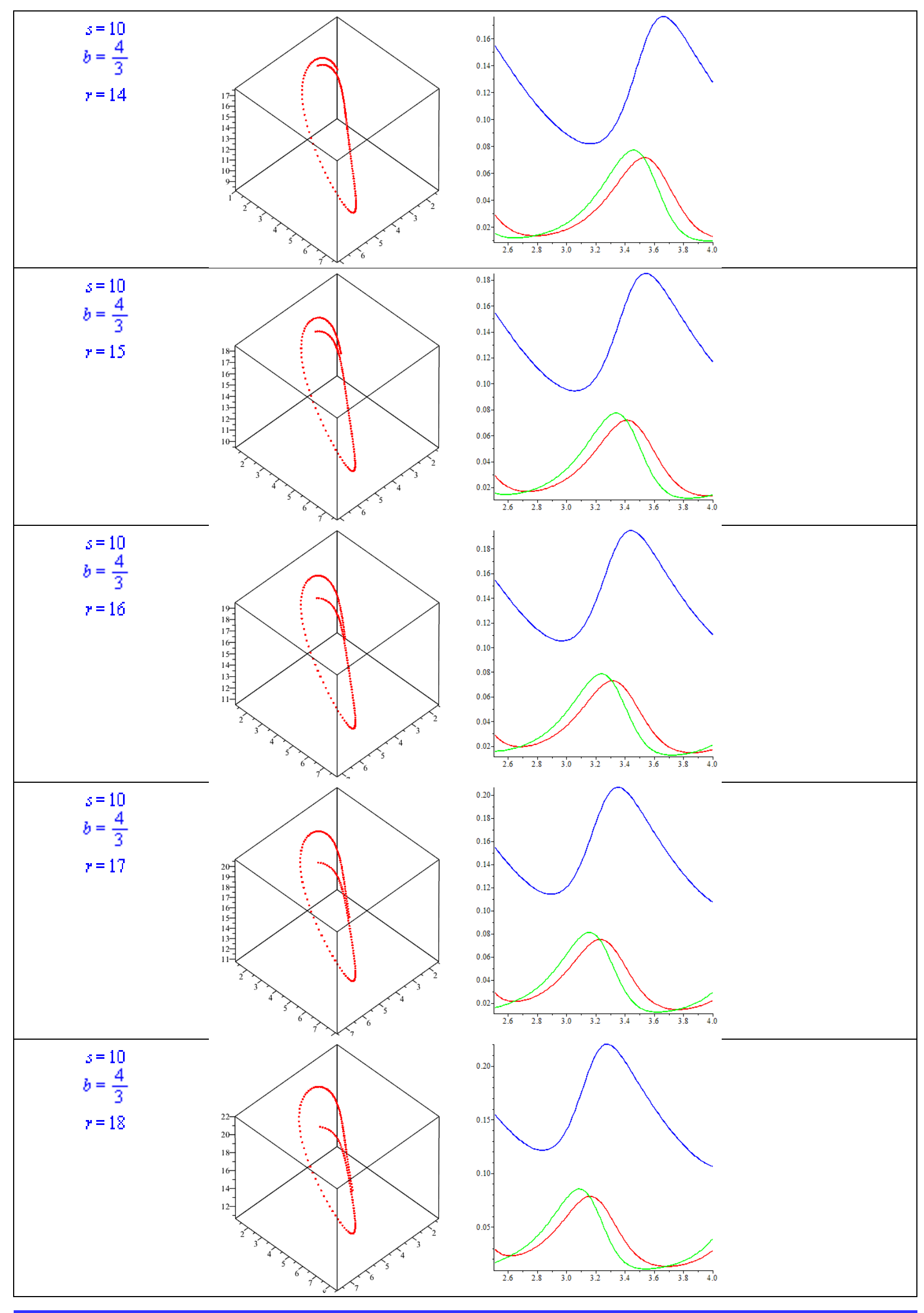




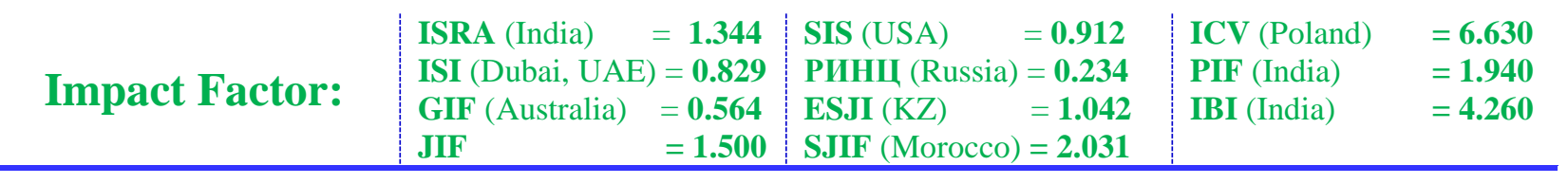

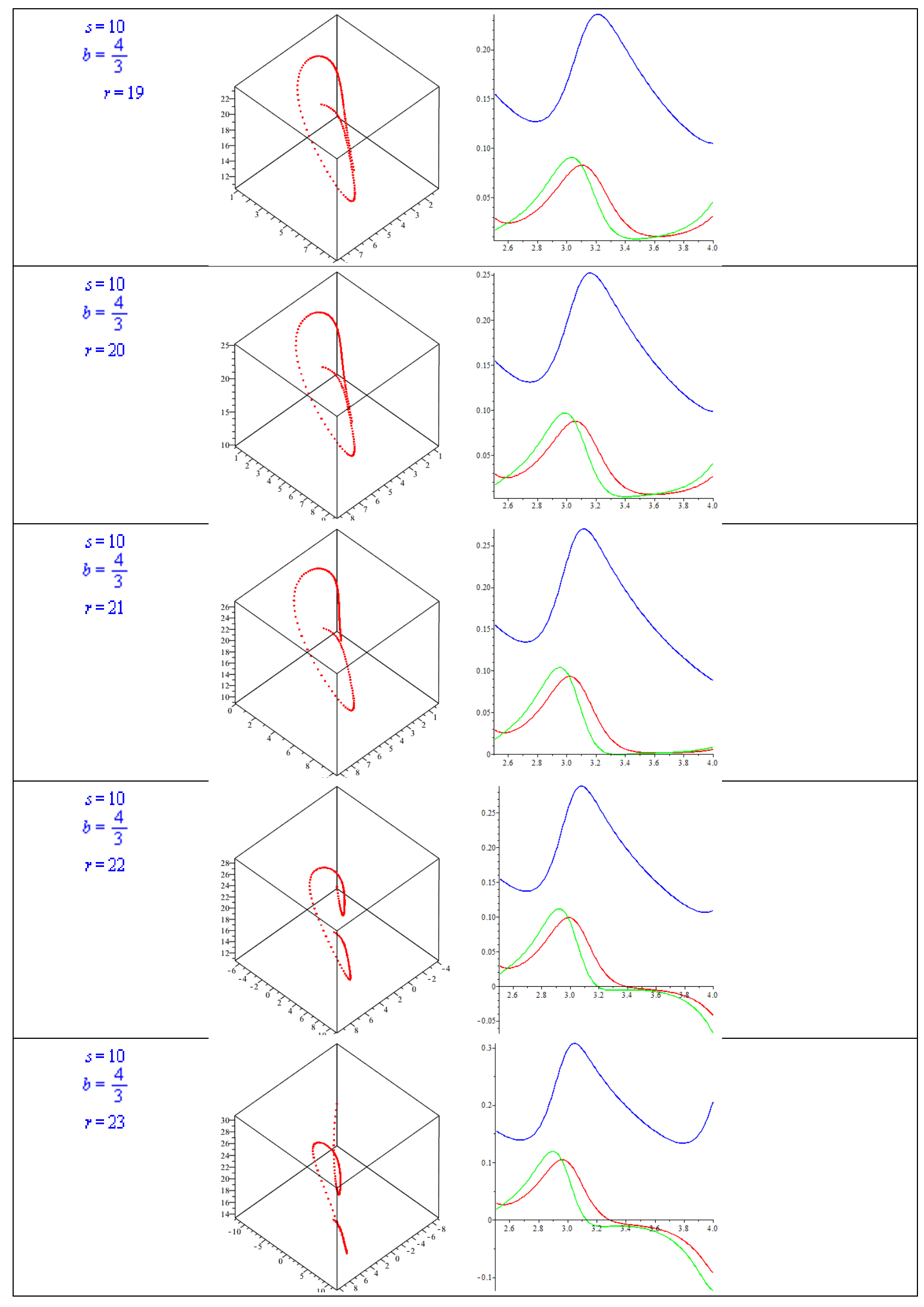




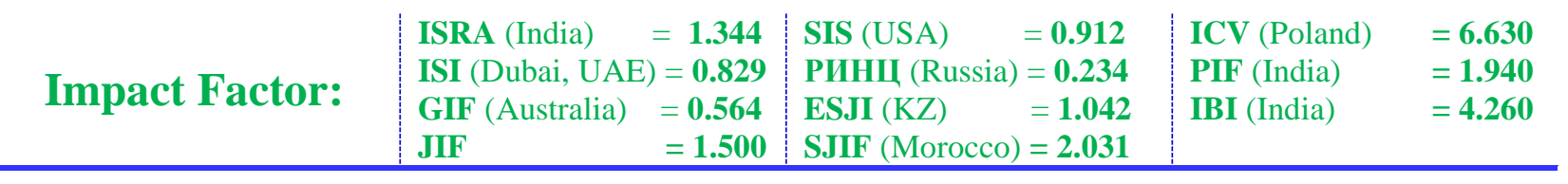

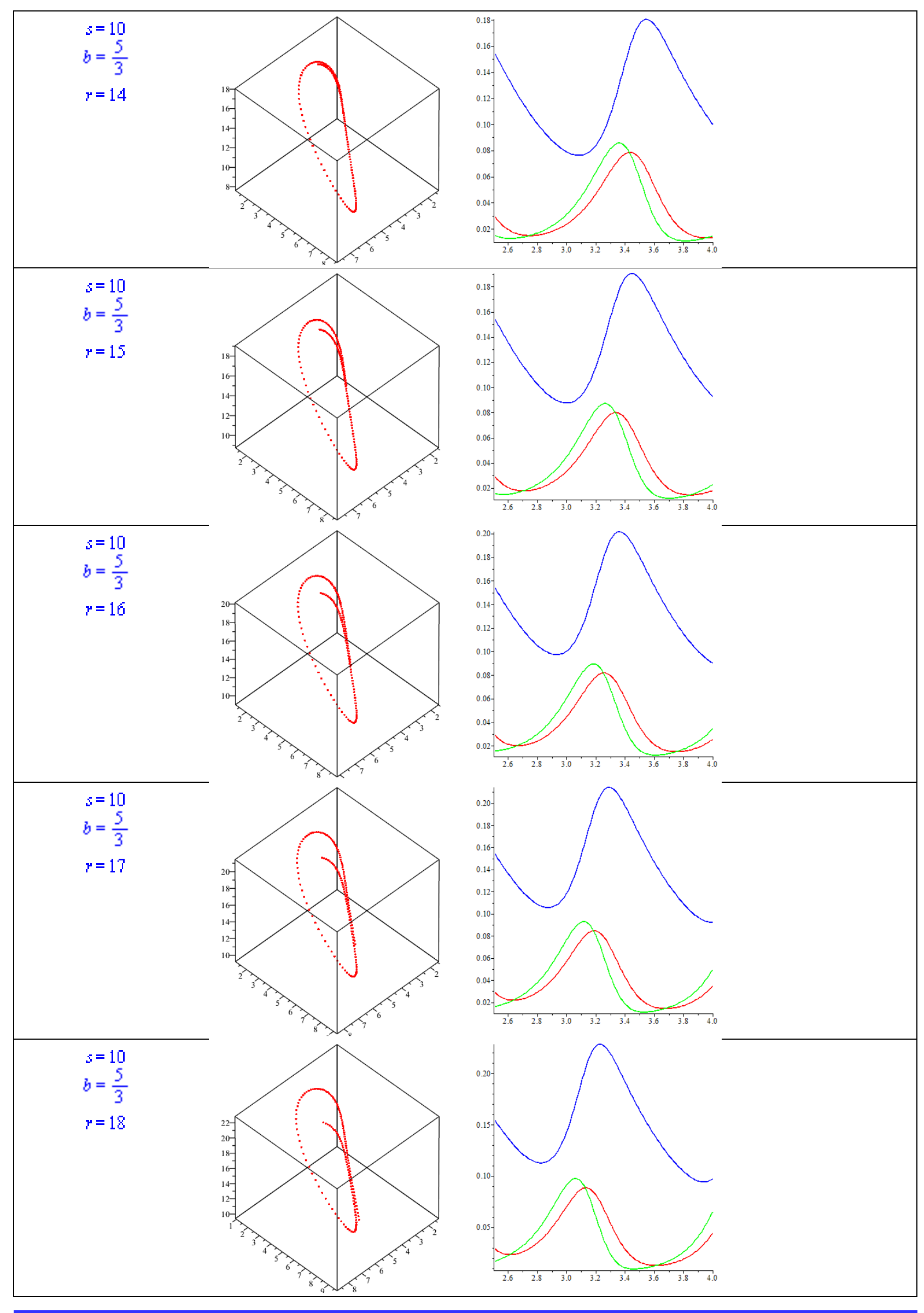




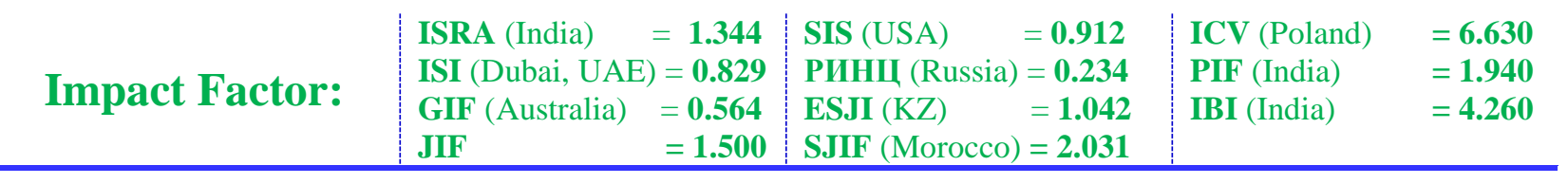

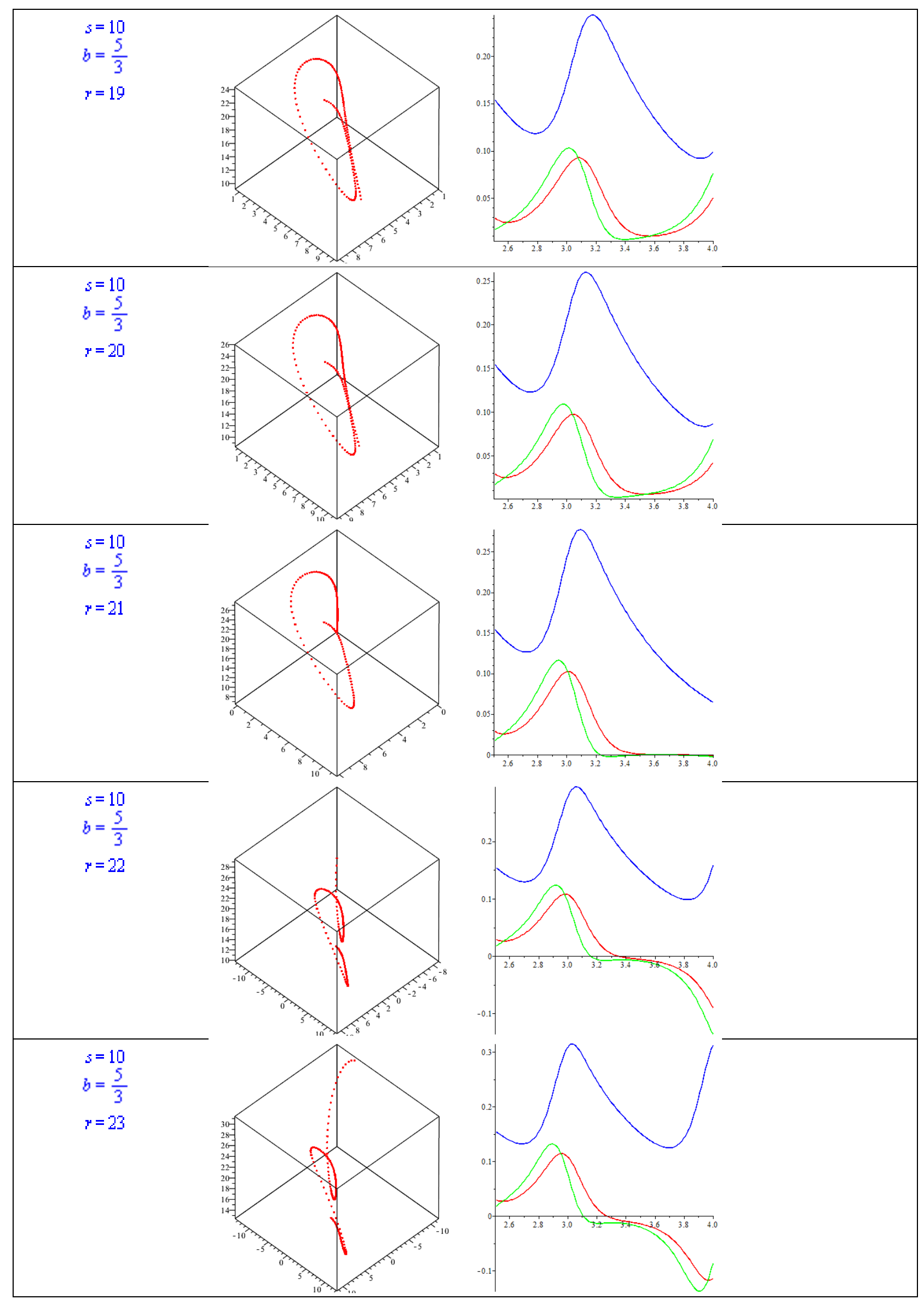




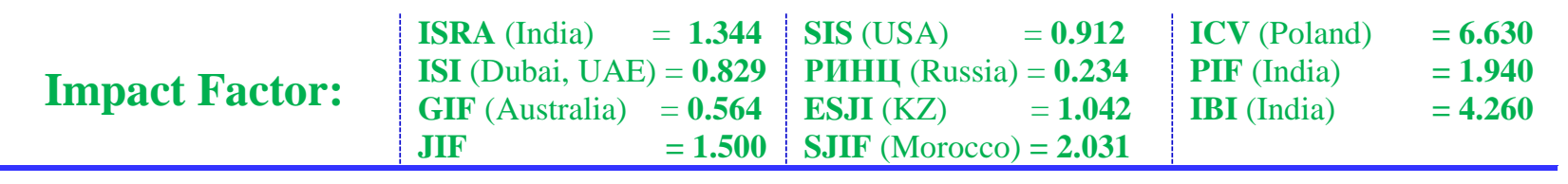

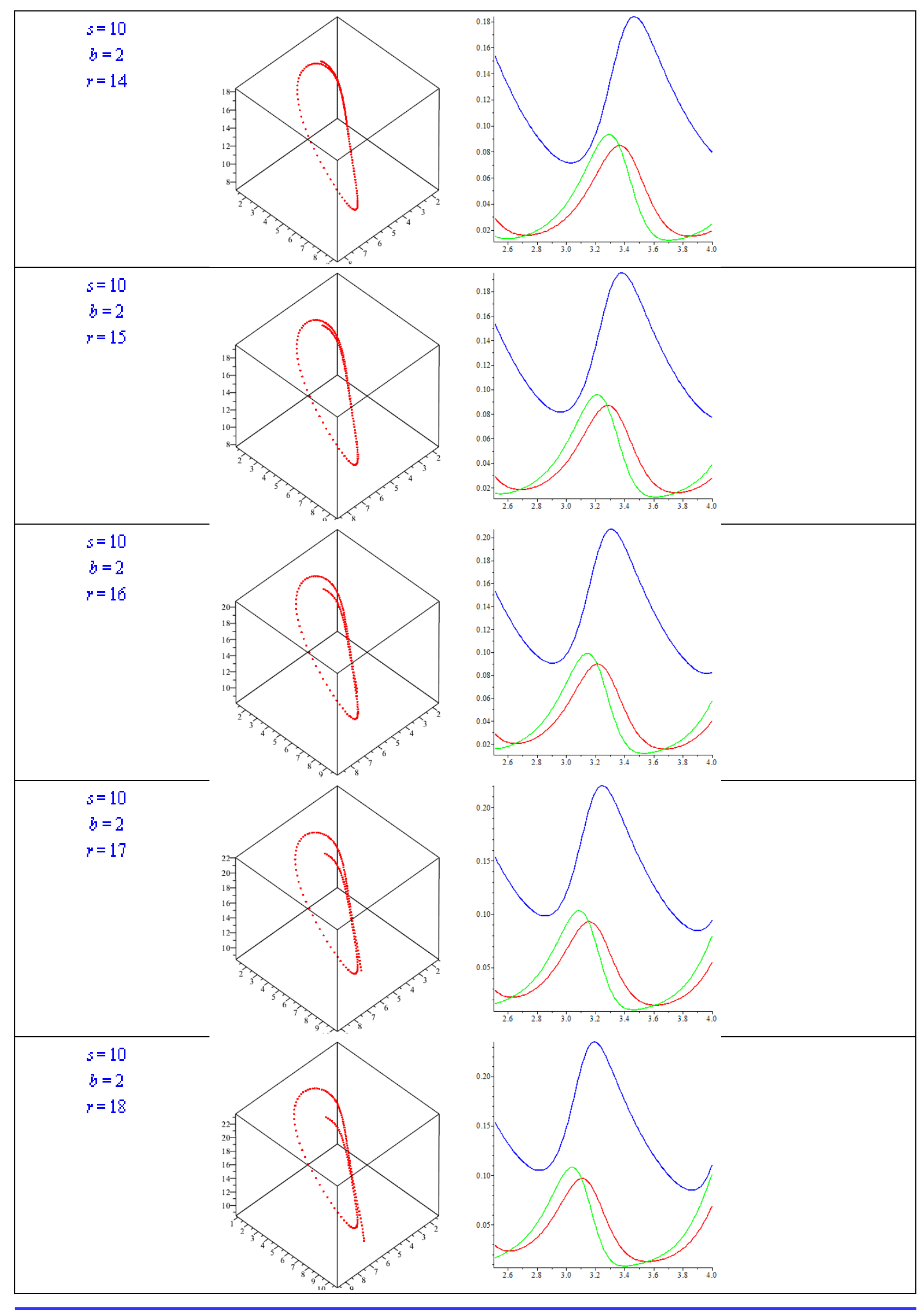




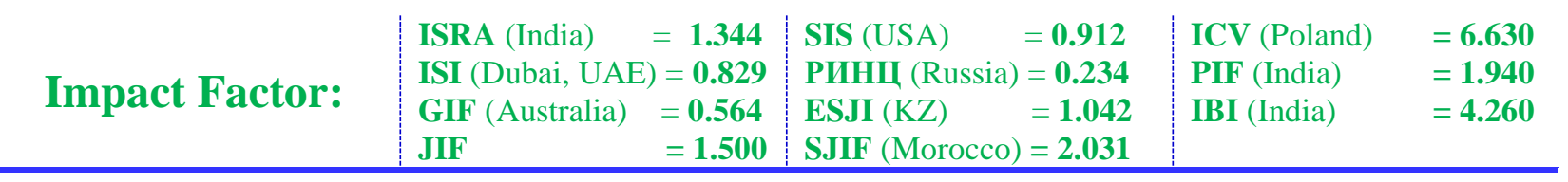

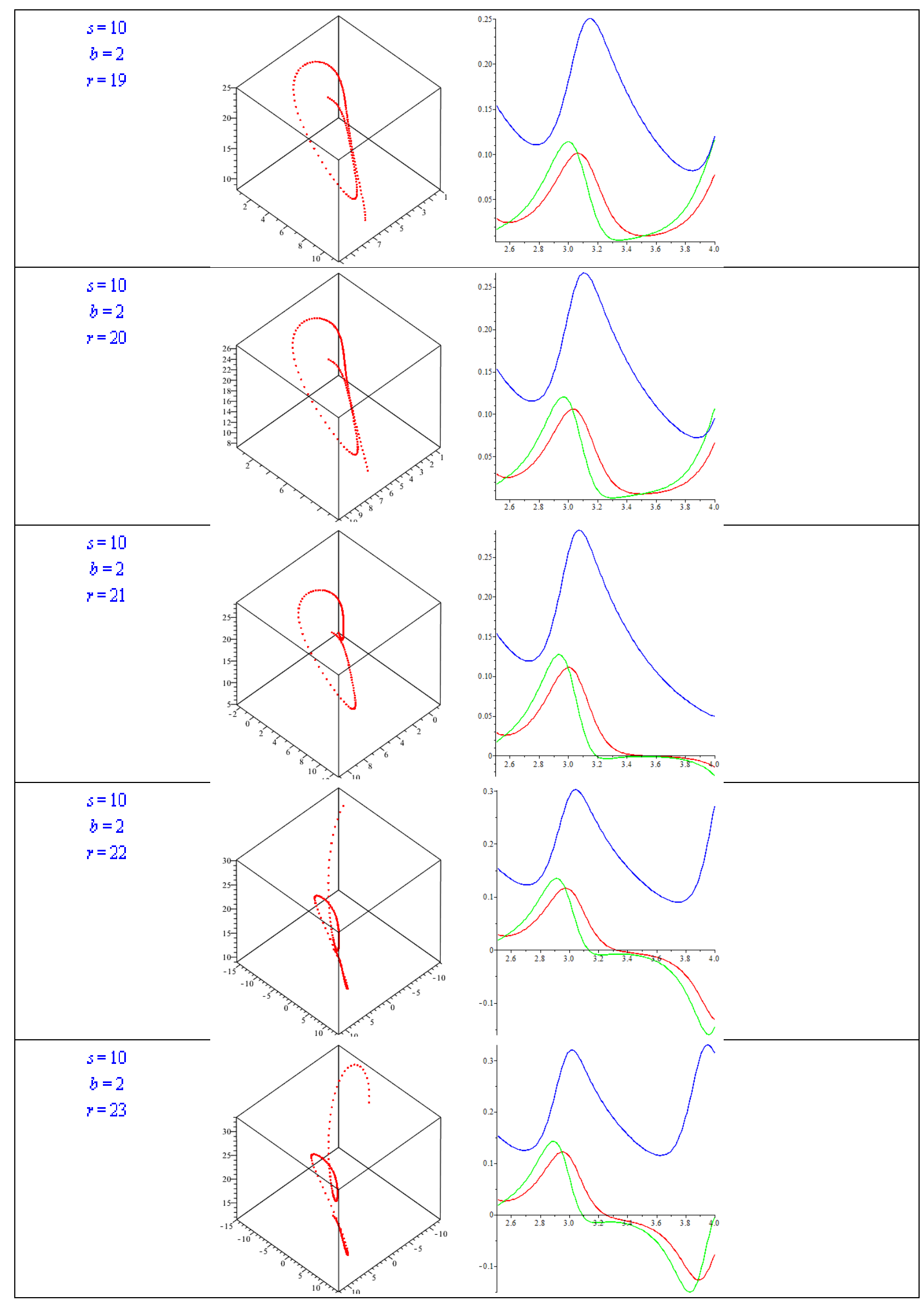




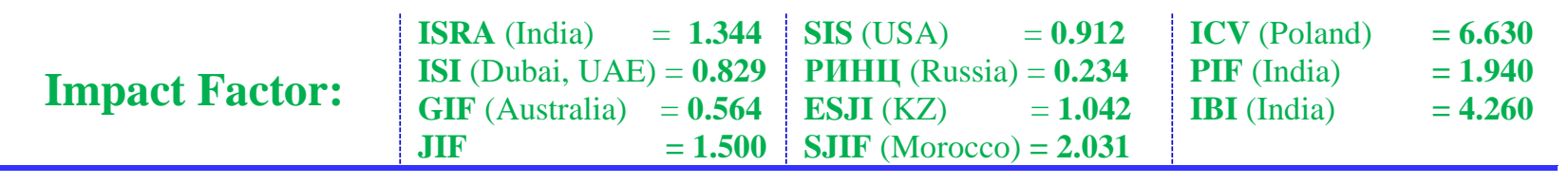

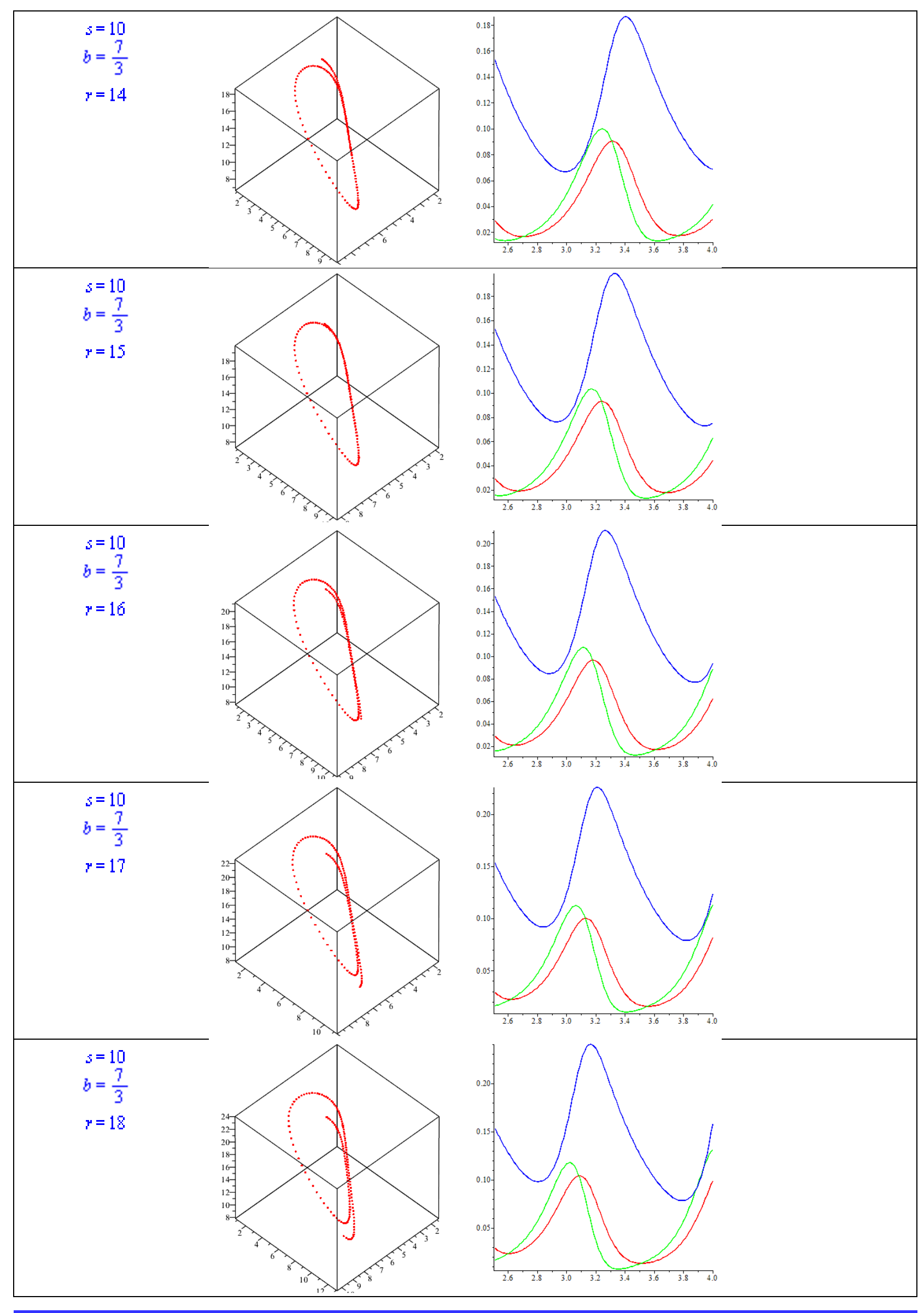




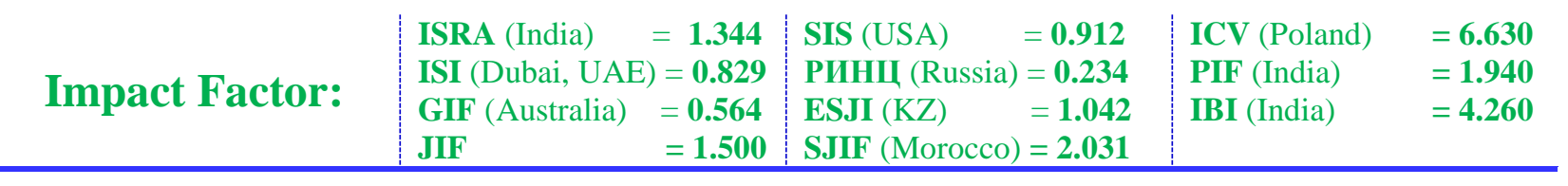

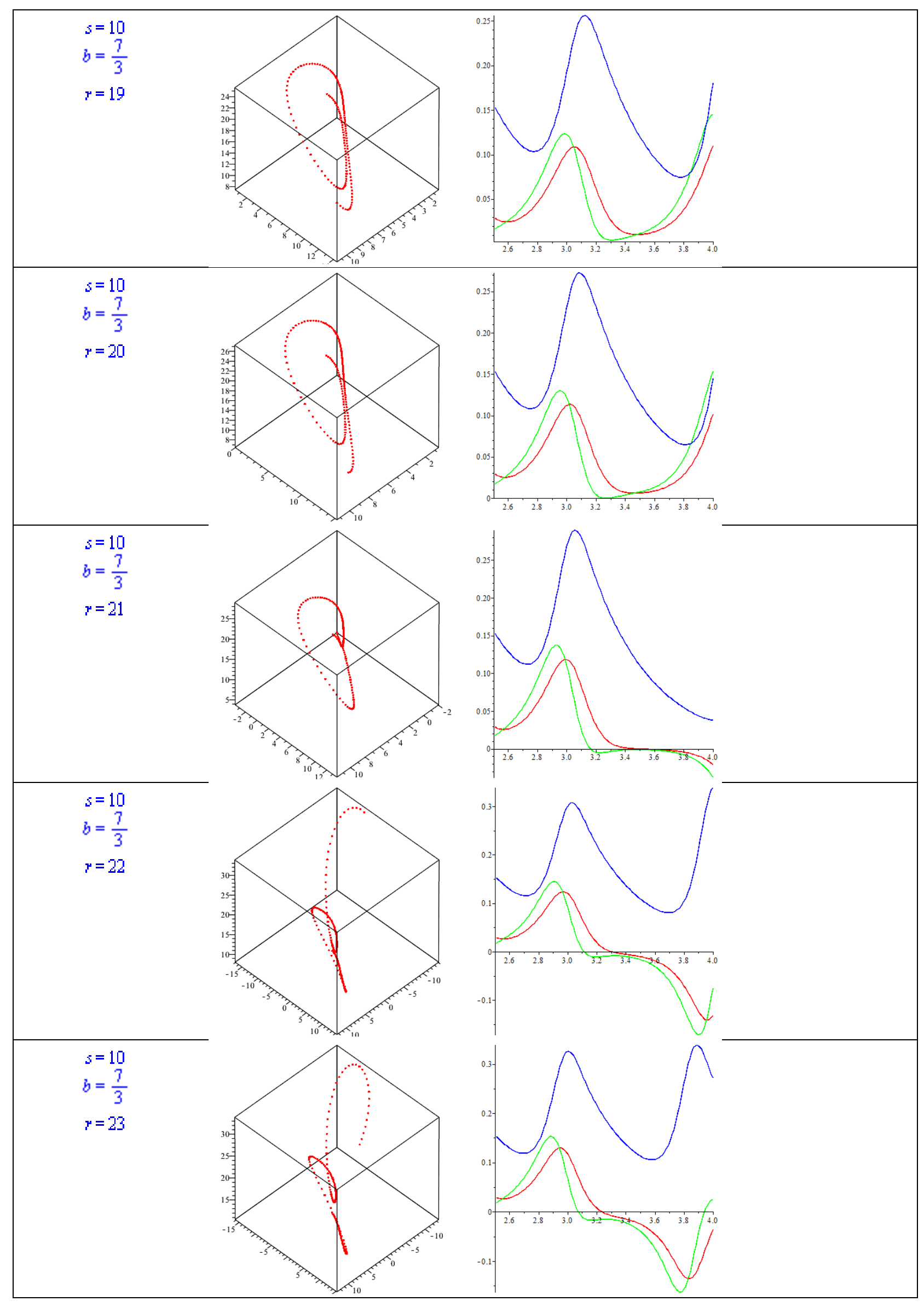




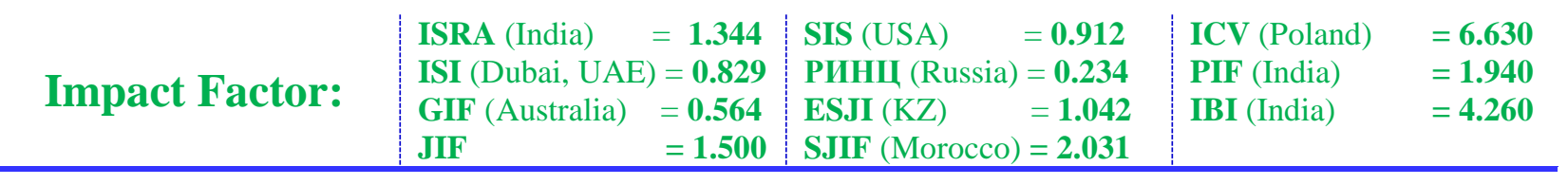

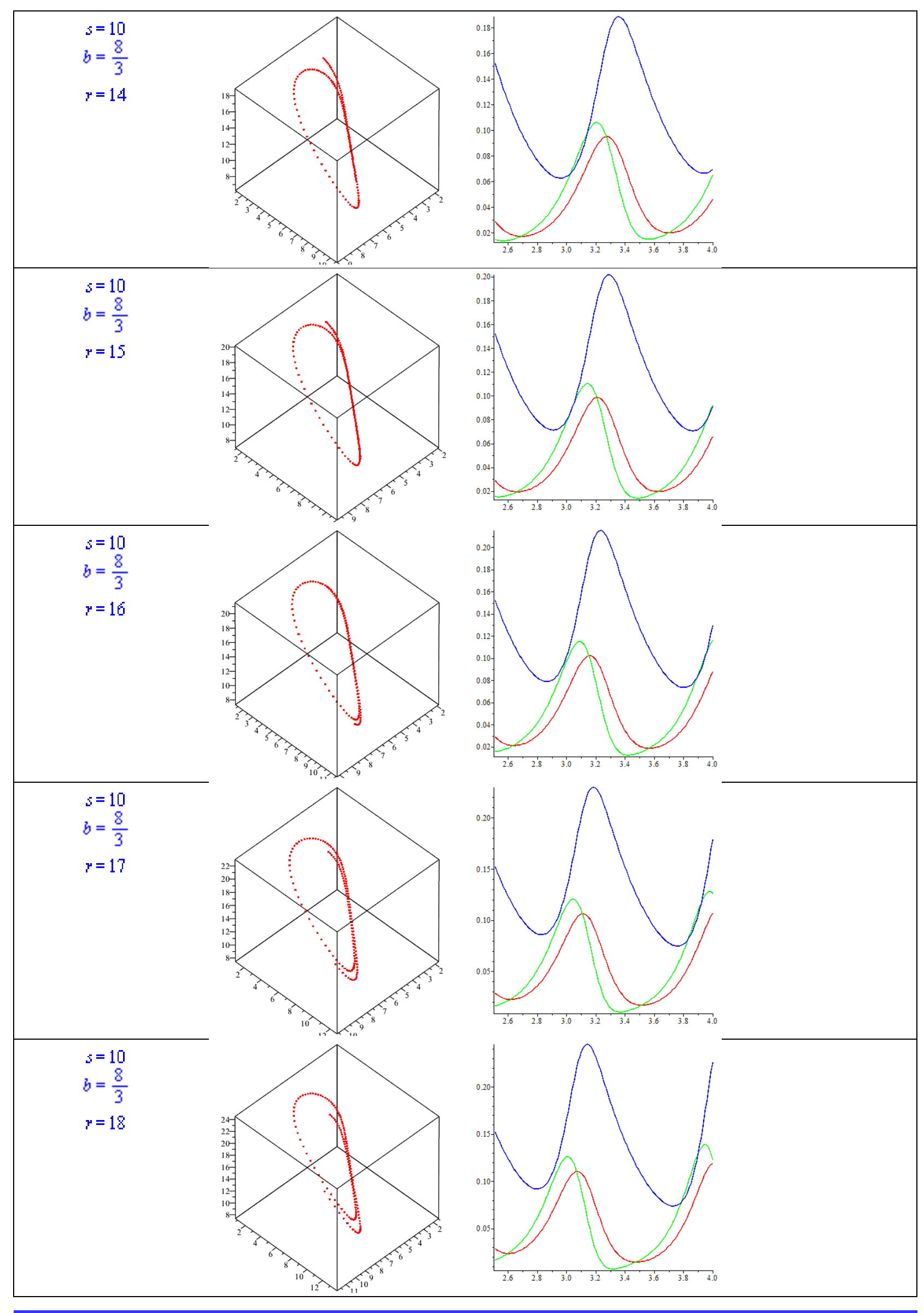




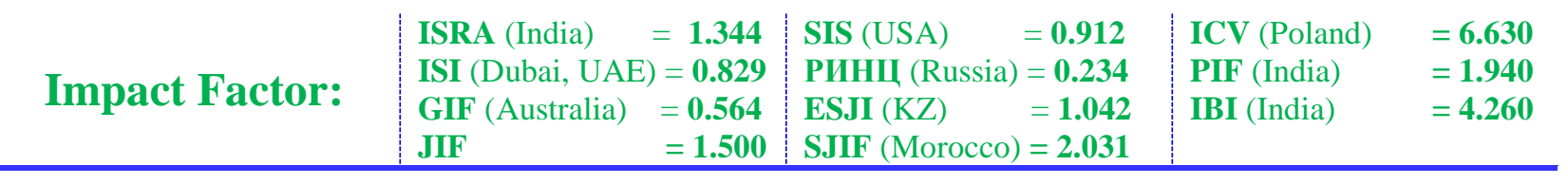

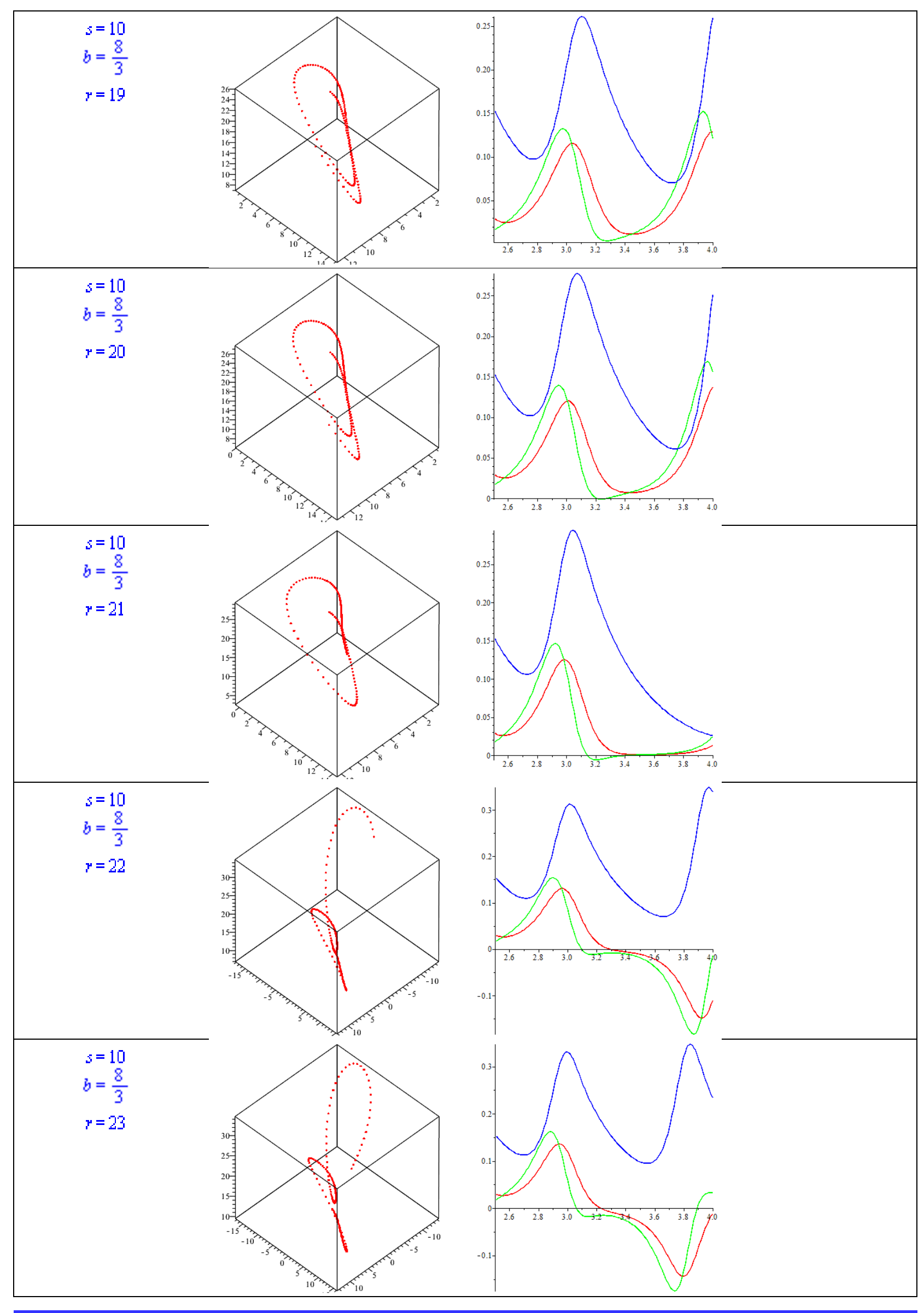




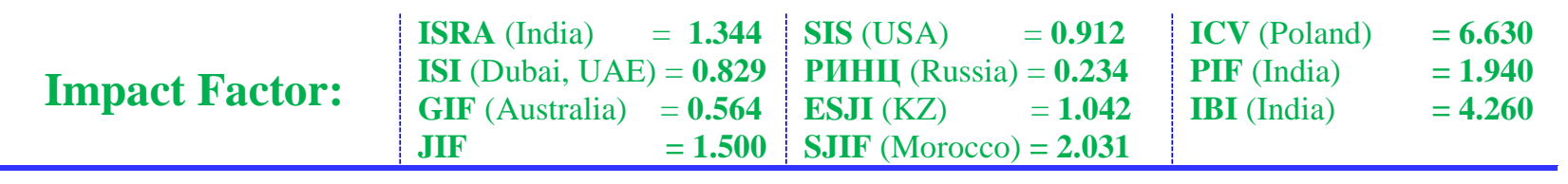

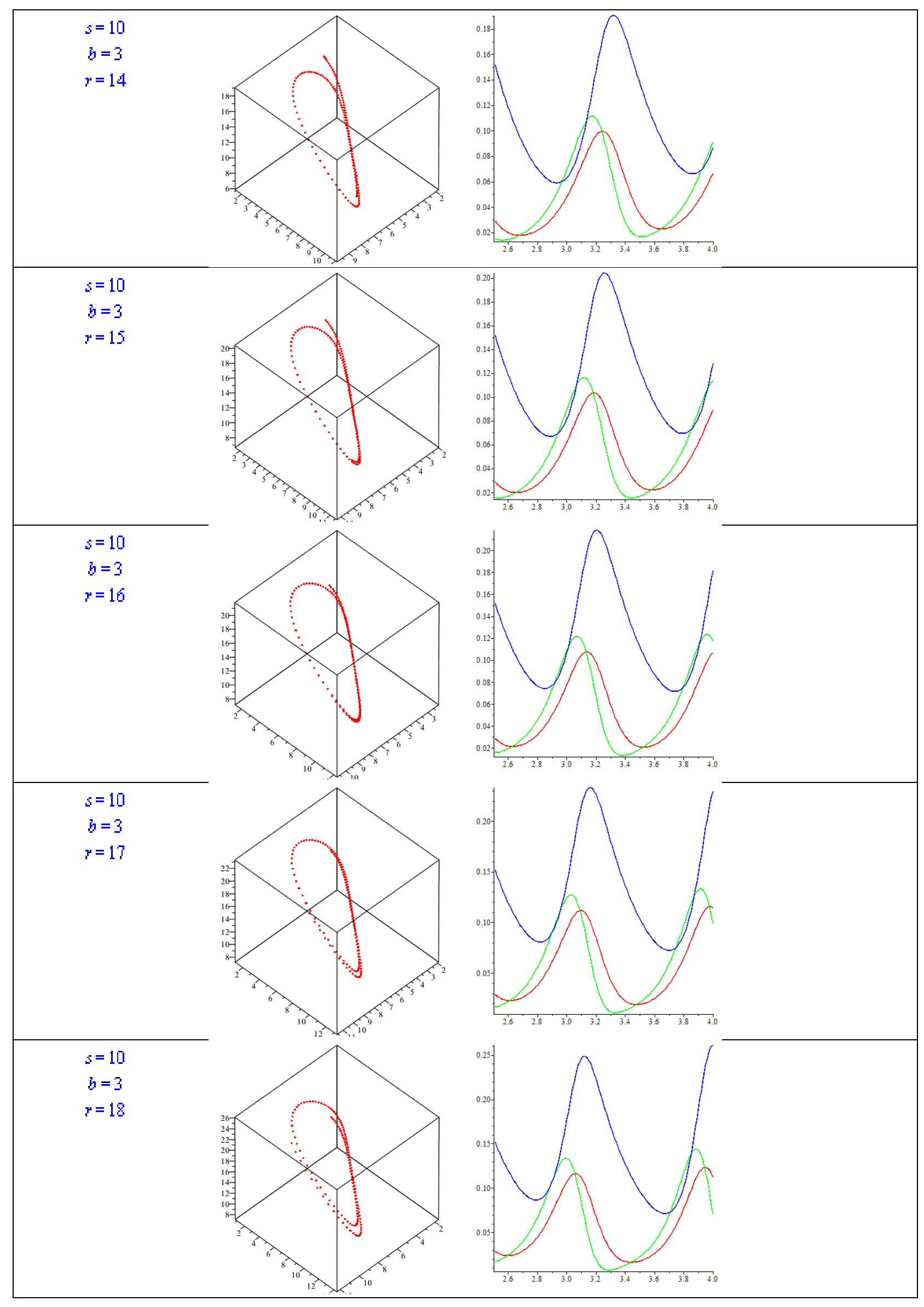




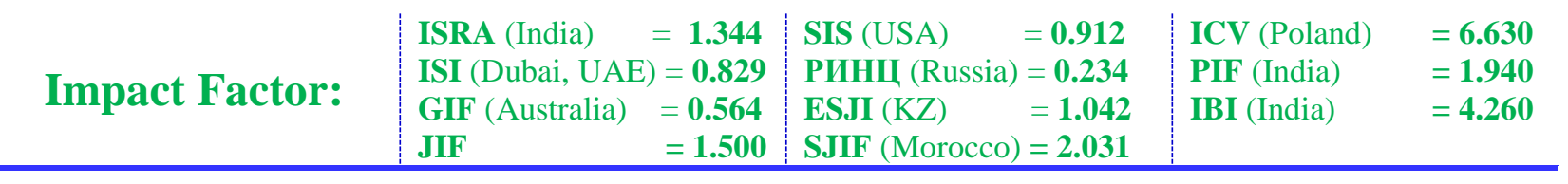

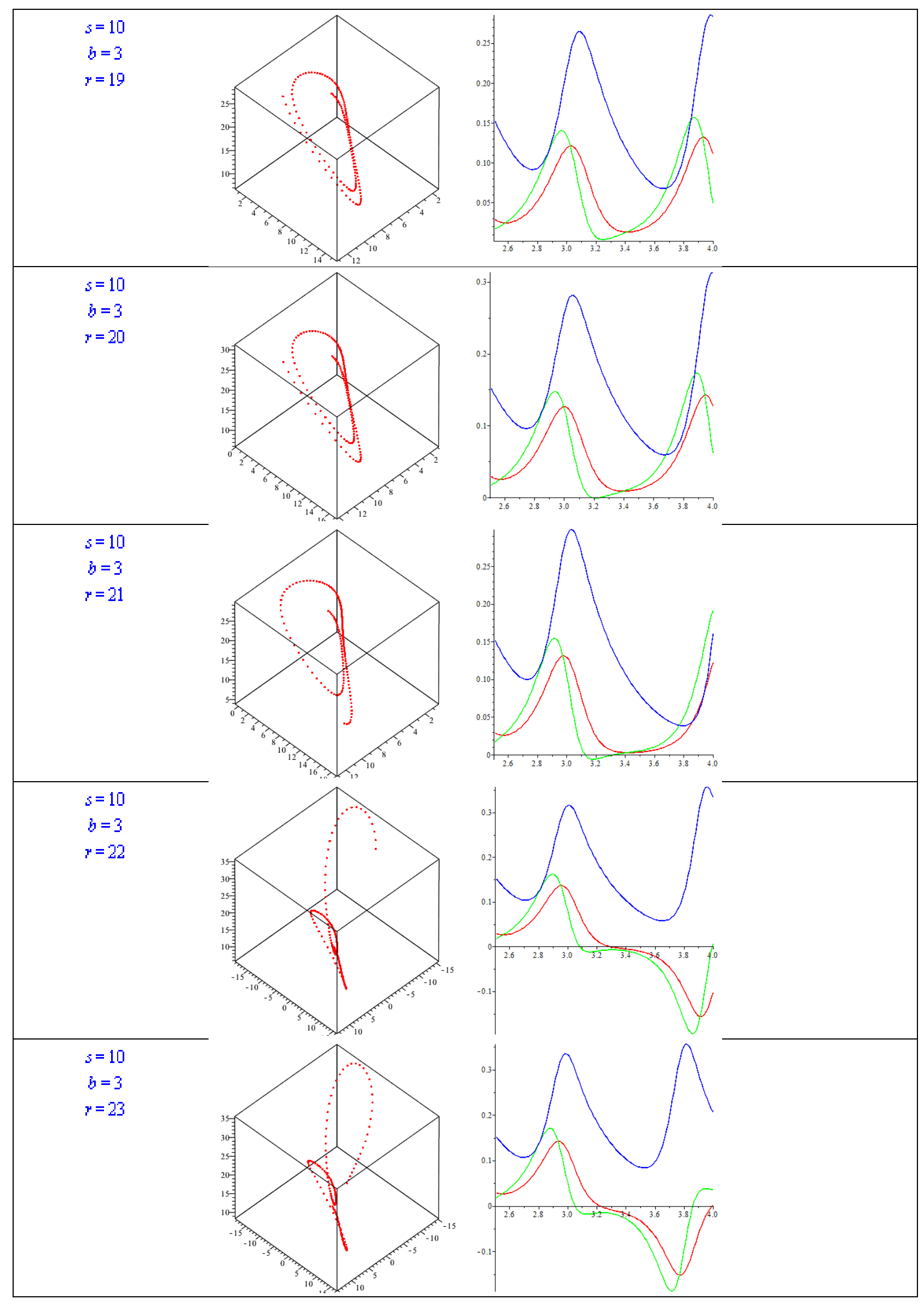




\begin{tabular}{l|lrl|l|ll} 
& ISRA (India) & $=\mathbf{1 . 3 4 4}$ & SIS (USA) & $=\mathbf{0 . 9 1 2}$ & ICV (Poland) & $=\mathbf{6 . 6 3 0}$ \\
Impact Factor: & ISI (Dubai, UAE) $=\mathbf{0 . 8 2 9}$ & PUHU (Russia) $=\mathbf{0 . 2 3 4}$ & PIF (India) & $=\mathbf{1 . 9 4 0}$ \\
& GIF (Australia) & $\mathbf{0 . 5 6 4}$ & ESJI (KZ) & $=\mathbf{1 . 0 4 2}$ & IBI (India) & $\mathbf{4 . 2 6 0}$
\end{tabular}

\section{Results}

The study of the bifurcation of the oscillation functions $\mathrm{x}, \mathrm{y}, \mathrm{z}$ for the Lorenz attractor for different values of the coefficients $b, r$.

\section{References:}

1. Sparrow S (1982) The Lorenz equations : Bifurcations, chaos and strange attractors. Springer Verlag, N. - Y. 1982.

2. Khaken G (1985) Sinergetika: ierarkhii neustoychivostey $\mathrm{v}$ samoorganizuyushchikhsya sistemakh i ustroystvakh. Moscow: Mir, 1985. $423 \mathrm{p}$.

3. Nikolis G, Prigozhin I (1979) Samoorganizatsiya v neravnovesnykh sistemakh. Moscow: Mir, 1979. 512 p.

4. Marpl-ml SL (1990) Tsifrovoy spektral'nyy analiz i ego prilozheniya. Moscow: Mir, 1990. $584 \mathrm{p}$.

5. Likhtenberg A, Liberman M (1984) Regulyarnaya i stokhasticheskaya dinamika. Moscow: Mir, 1984. 528 p.

6. Shuster G (1988) Determinirovannyy khaos. Vvvednie. Moscow: Mir, 1988. 240 p.
7. Gorley PN, Gorley PP, Tomchuk PM (1996) Pis'ma v ZhTF. 1996. T. 22. V. 20. pp. 82-86.

8. Korn G, Korn T (1970) Spravochnik po matematike dlya nauchnykh rabotnikov i inzhenerov. Moscow: Nauka, 1970. 720 p.

9. Williams RF (1979) The structure of the Lorenz attractors - Publ. Math. IHES, 1979, 50, pp. 321-347.

10. Yorke JA, Yorke ED (1979) Metastable chaos : the transition to sustained chaotic oscillations in a model of Lorenz - J. Stat. Phys., 1979, 21, pp. 263-267.

11. Rychlik M (1989) Lorenz attractors through a Shilnikov-type bufurcation, Part 1. Ergodic theory dynamical systems, 1989, 10, pp. 793821.

12. Tucker W (2002) A rigorous ODE solver and Smale's 14th problem -Found. Comput. Math., 2002, 2, pp. 53-117. 